\title{
CIT Burn Control Using Auxiliary \\ Power Modulation
}

\author{
E. A. Chaniotakis \\ J. P. Freidberg \\ D. R. Cohn \\ Massachusetts Institute of Technology \\ Plasma Fusion Center \\ Cambridge, MA, USA
}

29 September 1989 


\title{
CIT Burn Control Using Auxiliary Power Modulation
}

\author{
E. A. Chaniotakis
}

J. P. Freidberg

D. R. Cohn

Plasina Fusion Center

MIT

29 September 1989 


\section{Contents}

1 Introduction 2

2 Power Balance 2

3 Confinement Scalings 6

4 Ignition Definition $\quad \theta$

5 Tokamak thermal instability and the need for burn control 14

6 Mapping the Tokamak Operating Space 19

7 Burn Control Methods. 29

7.1 Passive Methods . . . . . . . . . . . . . . . 29

7.1.1 Soft $\beta$ Limit Control . . . . . . . . . . . . . 29

7.1.2 Field Ripple Control . . . . . . . . . . . . 30

7.2 Active Methods . . . . . . . . . . . . . 30

7.2 .1 Vary $B_{0} \ldots \ldots \ldots \ldots$

7.2.2 Adiabatic Expansion / Compression . . . . . . . 31

7.2.3 Vary the Plasma Density $n_{0} \ldots \ldots 31$

7.2.4 Vary the Auxiliary Power $P_{a} \ldots \ldots 32$

8 Burn Control Issues $\quad 34$

8.1 Thermal Equilibrium ................ 34

8.2 Stable Operation . . . . . . . . . . . . . . . 34

8.3 Time Scales . . . . . . . . . . . . . . . . . . 35

8.4 Diagnostic and Engineering lssues ........... 36

$9 \quad$ Auxiliary Power Control: General Remarks 37

10 The Complete Model 39

10.1 Time Delays . . . . . . . . . . . . . . . . . . 40

10.2 Density Evolution . . . . . . . . . . . . . . 41 
11 Results 44

11.1 Temperature Evolution . . . . . . . . . . . . . 444

11.2 Burn Control . . . . . . . . . . . . . . 52

12 Conclusions $\quad 63$ 


\section{Introduction}

It is predicted that ignited tokamaks such as CIT will experience a thermal instability. In this work a zero dimensional transport model is used as a means of examining this thermal instability. In order to solve the problems associated with the uncertainty in predicting the behavior of an ignited tokamak plasma, auxiliary power may be used for mapping the tokamak operating space, both in the low temperature stable regime and in the high temperature unstable regime. Auxiliary power modulation as a means of burn control is the most reliable method, especially during the initial stages of CIT operation. By modulating the auxiliary power stable thermal equilibria can be created at relatively high values of $Q(\sim 65)$.

\section{Power Balance}

In analyzing the burn control characteristics of a tokamak plasma the zero dimensional transport model will be used. Such a model describes the global power balance of the system and it is obtained by integrating the one dimensional transport equations over the plasma volume, and then substituting trial functions for the density and temperature profiles.

The general 0-D power balance of the ohmic $\left(p_{\Omega}\right)$, alpha particle $\left(p_{\alpha}\right)$, conduction loss $\left(p_{l}\right)$, Bremsstrahlung $\left(p_{b}\right)$ and auxiliary $\left(p_{a}\right)$ power densities has the form

$$
\left.{ }_{2}^{3}\left(n_{e}+n_{i}\right) \frac{\partial T}{\partial t}\right\rangle=\left\langle p_{n}\right\rangle+\left\langle p_{\alpha}\right\rangle+\left\langle p_{a}\right\rangle-\left\langle p_{l}\right\rangle-\left\langle p_{b}\right\rangle
$$

For a plasma composed of deuterium, tritium, alpha particles, and a single impurity with densities $n_{d}, n_{t}, n_{\alpha}$, and $n_{i m p}$ the following definitions are made

$$
\begin{aligned}
T & \equiv T_{e}=T_{D}=T_{T}=T_{i} \\
n_{i} & \equiv n_{d}+n_{t}+n_{\alpha}+n_{i m p} \\
n & \equiv n_{d}+n_{t} \\
f_{d} & \equiv \frac{n_{d}}{n}
\end{aligned}
$$


with $n$ representing the total deuterium-tritium density and $f_{d}$ gives the deuterium fractional density. Under these assumptions the power balance Eq. 1 takes the form

$$
\begin{aligned}
\left\langle\frac{3}{2}\left(n_{e}+n_{i}\right) \frac{\partial T}{\partial t}\right\rangle=\quad & \left\langle\eta J^{2}\right\rangle+\left\langle f_{d}\left(1-f_{d}\right) Q_{\alpha} n^{2} \bar{\sigma} v\right\rangle+\left\langle\frac{P_{a}}{V_{p}}\right\rangle- \\
& \left\langle\frac{3\left(n_{e}+n_{i}\right) T}{2 \tau_{E}}\right\rangle-\left\langle C_{b} n_{e}^{2} T^{1 / 2}\right\rangle
\end{aligned}
$$

where $\eta$ is the Spitzer resistivity [1], $J$ the current density, $Q_{\alpha}$ the alpha particle energy $(3.5 \mathrm{MeV}), \overline{\sigma v}$ the DT reaction rate, $P_{a}$ the total auxiliary power, $V_{p}$ the plasma volume, $\tau_{E}$ the energy confinement time, $C_{b}$ the Bremsstrahlung coefficient, and where $\langle\mathcal{F}\rangle$ denotes the average of $\mathcal{F}$ over the plasma volume. By charge neutrality the electron density is given by

$$
n_{\mathrm{e}}=n+2 n_{\alpha}+Z_{i m p} n_{i m p}
$$

The volume average of the terms in Eq. 6 is evaluated by assuming the plasma has an elliptic cross section with profiles given by

$$
\begin{aligned}
& n=n_{0}\left(1-\frac{x^{2}}{a^{2}}-\frac{y^{2}}{a^{2} \kappa^{2}}\right)^{\nu_{n}} \\
& T=T_{0}\left(1-\frac{x^{2}}{a^{2}}-\frac{y^{2}}{a^{2} \kappa^{2}}\right)^{\nu_{T}}
\end{aligned}
$$

Experimental measurements indicate that $\nu_{n} \approx 1 / 2$ for gas puffing while $\nu_{n} \approx 1$ for pellet injection. The quantity $\nu_{T}$ will soon be related to the kink safety factor $q_{*}[2]$ at the plasma edge. The on axis values $T_{0}$ and $n_{0}$ are related to the averages $\langle T\rangle$ and $\langle n\rangle$ by

$$
\begin{aligned}
& n_{0}=\left(\nu_{n}+1\right)\langle n\rangle \\
& T_{0}=\left(\nu_{T}+1\right)\langle T\rangle
\end{aligned}
$$


In this analysis we assume that all the density profiles are described by the same profile parameter $\nu_{n}$.

The ohmic power term is evaluated by assuming Spitzer resistivity [1] with $\ln \Lambda=16$.

$$
\eta=2.64 \times 10^{-8} Z_{\text {eff }} / T^{3 / 2} \quad \Omega m
$$

Here and in all formulae that follow the units are $T_{0}(\mathrm{keV}), n_{0}\left(10^{20} \mathrm{~m}^{-3}\right)$, $B_{0}(T), a(m), R_{0}(m)$ and $P_{a}(M W)$. The current density is related to the temperature by the steady state ohmic relation

$$
J=J_{0}\left(T / T_{0}\right)^{3 / 2}
$$

where the current density on axis $J_{0}$ is determined by the sawtooth condition $q_{0}=1$.

$$
J_{0}=\left(\frac{1+\kappa^{2}}{\kappa}\right)\left(\frac{B_{0}}{\mu_{0} R_{0} q_{0}}\right)
$$

Eq. 13 is assumed valid for $P_{a}=0$ as well as $P_{a} \neq 0$ in order to avoid the unrealistically optimistic situation of simultaneously peaked temperature profiles and broad current profiles.

The profile parameter $\nu_{T}$ can now be related to the kink safety factor $q_{*}$ by the definition [2]

$$
q_{*} \equiv \frac{2 \pi a^{2} \kappa B_{0}}{\mu_{0} R_{0} I}
$$

From Eqs. 13 and 14 and by using the relation $I=\int J d A$ we obtain

$$
\frac{q_{*}}{q_{0}}=\frac{2 \kappa}{1+\kappa^{2}}\left(1+1.5 \nu_{T}\right)
$$

The physical significance of Eq. 16 is that as the total current increases the temperature profile becomes broader. 
Finally, the ohmic power term is given by

$$
\left\langle p_{\Omega}\right\rangle=\frac{1.67 \times 10^{4} Z_{\text {eff }}}{1+1.5 \nu_{T}}\left(\frac{1+\kappa^{2}}{\kappa}\right)^{2} \frac{B_{0}^{2}}{q_{0}^{2} R_{0}^{2} T_{0}^{3 / 2}} \quad W / m^{3}
$$

The alpha power density is given by

$$
\left\langle p_{\alpha}\right\rangle=\frac{5.6 \times 10^{5}}{\nu_{T}} f_{d}\left(1-f_{d}\right) n_{0}^{2} F_{\alpha}\left(T_{0}\right) \quad W / m^{3}
$$

where

$$
F_{\alpha}\left(T_{0}\right)=\frac{10^{22}}{T_{0}{ }^{\bar{\gamma}}} \int_{0}^{T_{0}} \overline{\sigma v}(\xi) \xi^{\gamma-1} d \xi
$$

with $\overline{\sigma v}$ given in $m^{3} / \sec$ and $\gamma \equiv\left(2 \nu_{n}+1\right) / \nu_{T}$.

Two types of confinement scalings are considered. The ohmic (NeoAlcator) [3] scaling $\tau_{N A}$, and the auxiliary scaling $\tau_{A U}$. Since the ohmic scaling represents an upper limit on confinement a combination of ohmic and auxiliary scalings of the form [4]

$$
\begin{aligned}
\frac{1}{\tau_{E}} & =\left(-\frac{1}{\tau_{N A}^{2}}+\frac{1}{\tau_{A U}^{2}}\right)^{1 / 2} \\
& =\frac{1}{\tau_{N A}}\left(1+\Lambda^{2}\right)^{1 / 2}
\end{aligned}
$$

is used in order to limit $\tau_{E}$. The form of the ohmic scaling considered as well as various forms of the auxiliary scalings are given in the next section. The thermal conduction losses are given by

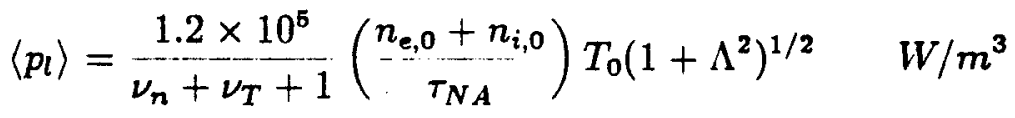


where $\Lambda \equiv \tau_{N A} / \tau_{A U}$, and $n_{\varepsilon, 0}, n_{i, 0}$ are the peak values of the electron and ion densities.

The average auxiliary power heating density is defined as the total power absorbed by the plasma, $P_{a}$, divided by the plasma volume. Thus

$$
\left\langle p_{a}\right\rangle=\frac{P_{a}}{2 \pi^{2} a^{2} R_{0} \kappa}=5.07 \times 10^{4}\left(\frac{P_{a}}{a^{2} R_{0} \kappa}\right) \quad W / m^{3}
$$

Finally the radiative loss due to Bremsstrahlung is given by

$$
\left\langle p_{b}\right\rangle=\frac{5.3 \times 10^{3} Z_{e f f}}{2 \nu_{n}+.5 \nu_{T}+1} n_{e, 0}^{2} T_{0}^{1 / 2} \quad W / m^{3}
$$

Eqs. 17, 18, 21, 22, and 23 are now substituted in Eq. 6 and the power balance becomes

$$
\begin{gathered}
K_{l}\left(n_{e}+n_{i}\right) \dot{T}=\quad K_{n} \frac{B^{2}}{q_{0}^{2} R^{2} T^{3 / 2}}+K_{\alpha} f_{d}\left(1-f_{d}\right) n^{2} F_{\alpha}(T)-K_{b} n_{e}^{2} T^{1 / 2}- \\
K_{l} \frac{\left(1+\Lambda^{2}\right)^{1 / 2}}{\tau_{N A}}\left(n_{e}+n_{i}\right) T+K_{a} P_{a}
\end{gathered}
$$

where the zero subscript has been suppressed from $T_{0}, B_{0}, R_{0}, n_{e, 0}, n_{i, 0}, n_{0}$, and $\dot{T} \equiv \partial T / \partial t$. The parameters $K_{j}$ are given in Appendix A.

\section{Confinement Scalings}

The energy confinement scalings play two important roles in an ignition experiment. First, at the design stage it gives information on the values of the machine parameters $R, a, R / a, B, \kappa, I$ etc. needed in order to achieve the design goal. Second, during the operation stage the confinement characteristics are used in order to predict the behavior of a given shot.

The first goal is achieved by using the existing confinement scalings with the appropriate extrapolations in the regime of interest. This approach does 
not guaranty success since it is only capable of predicting the behavior in the regime from which the data base has been extracted. It is however hoped that the design will provide a window for achieving the desired goal. The goal of predicting the exact behavior during operation will be met by performing experiments designed to investigate the new regimes of interest in a controlled fashion. In achieving this goal we propose the use of auxiliary power modulation for burn control and will discuss the method in detail in later sections. In the analysis that follows we will use the energy confinement of the form given by Eq. 20 and will investigate both ohmic and auxiliary heated confinement.

Ohmic confinement is obtained when ohmic heating represents the primary heating mechanism. The most well known ohmic scaling is the NeoAlcator scaling [3] and its effect can be investigated by setting $\Lambda=0$ in the energy balance Eq. 24.

Confinement in auxiliary heated tokamaks appears to degrade with power [4] and various scalings exist representing this mode of operation. In this paper we will not analyze the tokamak behavior under all these scalings. Rather we will concentrate on the efferts of the Goldston scaling, one of the most widely used auxiliary power scalings. In the following analysis we assume that alpha power degrades confinement in the same manner that auxiliary power has been observed to degrade confinement. The Goldston scaling is given by [4]

$$
\tau_{G L}=0.037 M \frac{I_{p} \kappa^{.5} R^{1.75} A^{.5}}{P .5} a^{.37} \quad \sec
$$

where again $I_{p}$ is given in $\mathrm{MA}, P=P_{a}+P_{\alpha}$ is given in $\mathrm{MW}, \mathrm{M}$ is a multiplicative factor measuring possible enhancements due to $\mathrm{H}$ mode operation, and $A=5 / 3$ represents the normalized mass ratio for $\mathrm{D}-\mathrm{T}$ reactions. The energy confinement time has the form

$$
\frac{1}{\tau_{E}}=\frac{1}{\tau_{N A}}\left(1+\Lambda_{G L}^{2}\right)^{1 / 2}
$$


where $\Lambda_{G L}=\tau_{N A} / \tau_{G L}$ reduces to

$$
\Lambda_{G L}=K_{G L} N \Gamma^{.5}
$$

$\Gamma$ and $K_{G L}$ are given by:

$$
\begin{gathered}
\Gamma=C_{1} \frac{R P_{a}}{a}+C_{2}\left(\frac{n_{e} R}{B}\right)^{2} B^{2} a F_{\alpha} \\
K_{G L}=\frac{3.71}{M f} \frac{q_{*}}{R^{.25} a^{.13} \kappa^{.75}}
\end{gathered}
$$

$F_{\alpha}$ is given by Eq. 19 and $C_{1}, C_{2}$, and $f$ are given in Appendix A. The behavior under Goldston scaling is thus obtained by substituting Eq. 27 into Eq. 24. 


\section{Ignition Definition}

A tokamak thermonuclear plasma will be considered ignited once the plasina temperature reaches a point beyond which the plasma temperature increases mainly due to heating by the alpha particles.

The ignition definition used herein follows from an examination of a $\dot{T}$ vs $T$ plot such as the one shown in Fig. 1. Note that for $P_{a}=0$ there are, in general, three steady state equilibrium solutions corresponding to $\dot{T}=0$. The left equilibrium point, denoted by $T_{\Omega}$, represents the temperature achieved in an ohmic discharge $\left\langle p_{n}\right\rangle \approx\left\langle p_{l}\right\rangle$ and it is dominated by the $1 / T^{3 / 2}$ dependance of the ohmic power term. The equilibrium point $T_{B}$, for burn temperature, is dominated by the alpha particle heating and occurs in the range of $10-20$ $\mathrm{keV}$. Since the temperature for maximum fusion power production, at fixed $\beta$, occurs at $\sim 15 \mathrm{keV}$ it is desired that future fusion reactors operate in this temperature regime.

The right equilibrium point, $T_{R}$, is a consequence of the high temperature decay of the $\overline{\sigma v}$ curve and it typically occurs between $30-90 \mathrm{keV}$, depending on the particular tokamak design and the energy confinement scaling used.

The temperatures at which an ignition experiment is expected to operate are shown by the shaded region in Fig. 1. However, it is not possible to gain access to these high temperatures through the normal evolution of an ohmic discharge. This is because $T_{\Omega}$ is a point of stable operation. Therefore, in order to reach the high temperature regime the minimum of the $\dot{T}$ curve must lie above the axis. This may be achieved with the use of auxiliary power. Once the minimum of the $\dot{T}$ curve becomes greater than zero the alpha power dominates the energy balance and the plasma temperature increases due to self heating by the alpha particles. The temperature at which the minimum of the $\dot{T}$ curve becomes tangent to the axis is denoted by $T_{I}$, and will be called Marginal Ignition Temperature hereinafter. Fig. 2 shows a $\dot{T}$ vs $T$ plot for 


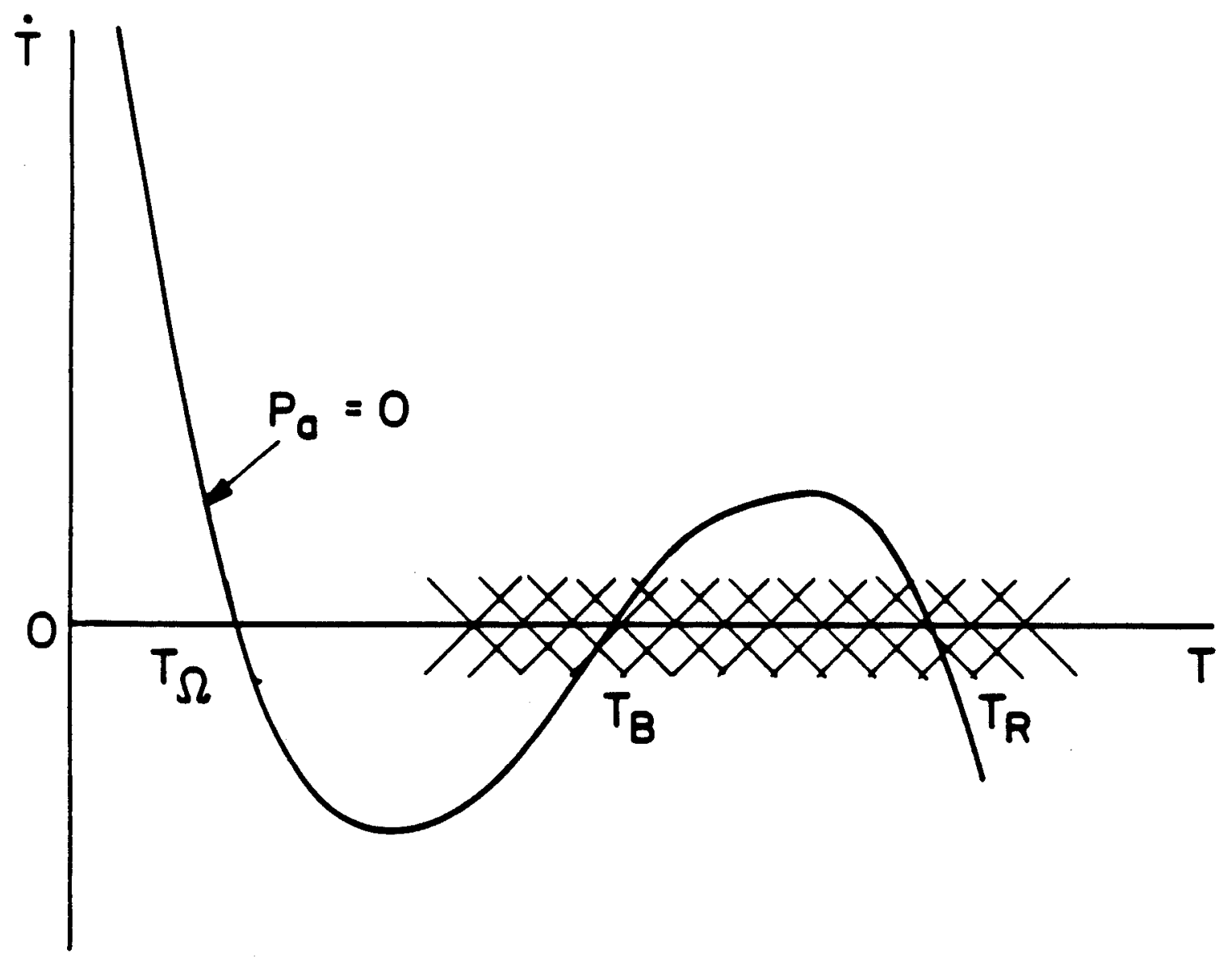

Figure 1: Plot of the rate of change of temperature with time, $\dot{T}$, vs temperature $T$. 


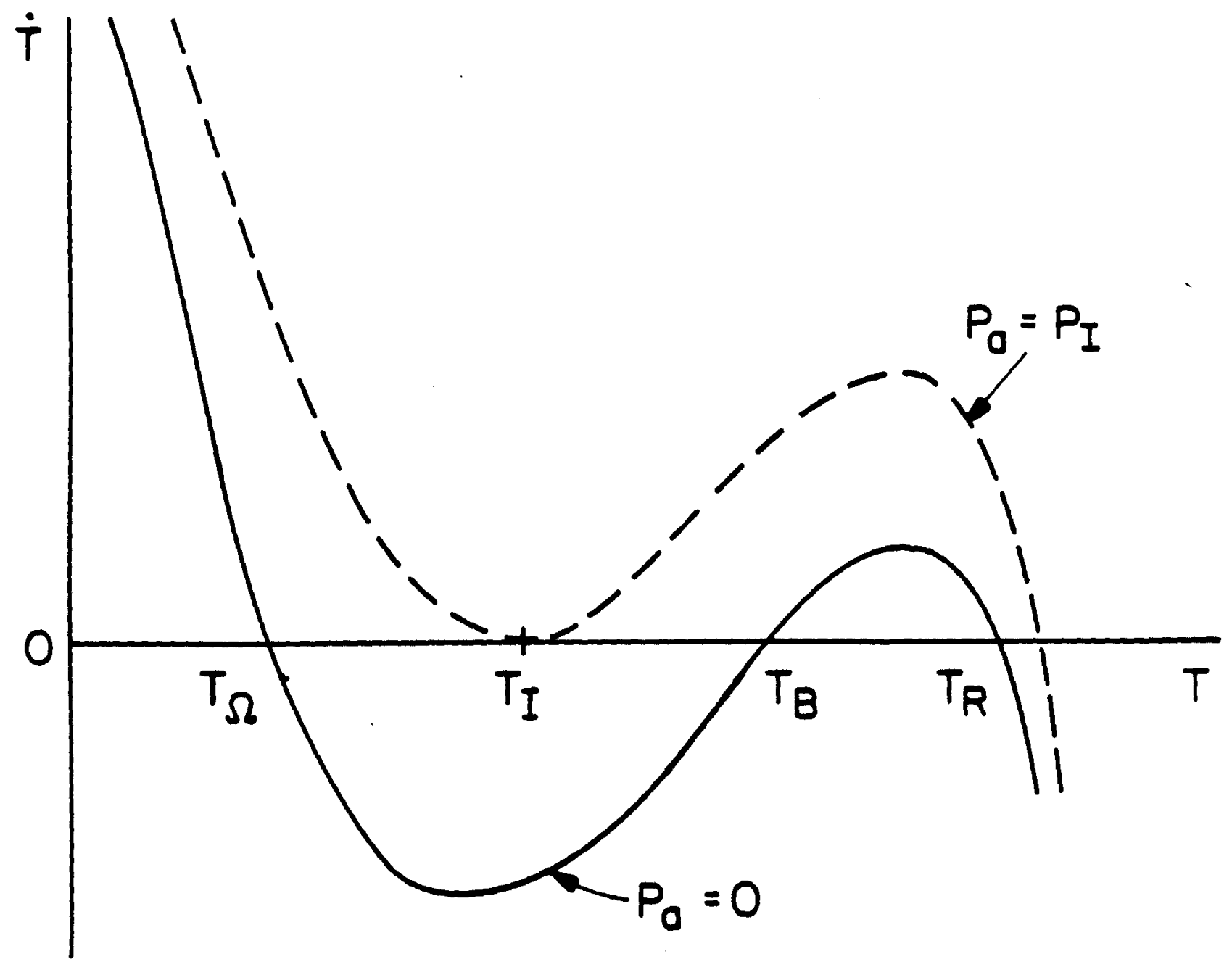

Figure 2: A plot showing the original system (solid line) and the ignited system with $P_{a}=P_{I}$ (dotted line). 
zero auxiliary power, $P_{a}=0$, (solid line) and for $P_{a}=P_{I}$ (dotted line), where $P_{I}$ is the amount of auxiliary power required to make the minimum of the $\dot{T}$ vs $T$ plot tangent to the axis at $T_{I}$. It should be noted that $P_{I}$ is the auxiliary power absorbed by the plasma.

On an n-T (POPCON) plot the marginal ignition temperatures form a contour as shown in Fig. 3. This contour goes through the Cordey pass and will be referred to as the Marginal Ignition Ridge (MIR) hereinafter.

A plasma will thus be considered ignited only if the temperature exceeds that corresponding to the MIR. Mathematically the conditions for marginal ignition described above is given by [5]

$$
\begin{aligned}
\dot{T}\left(T_{I}\right) & =0 \\
\frac{\partial \dot{T}}{\partial T}\left(T_{I}\right) & =0
\end{aligned}
$$




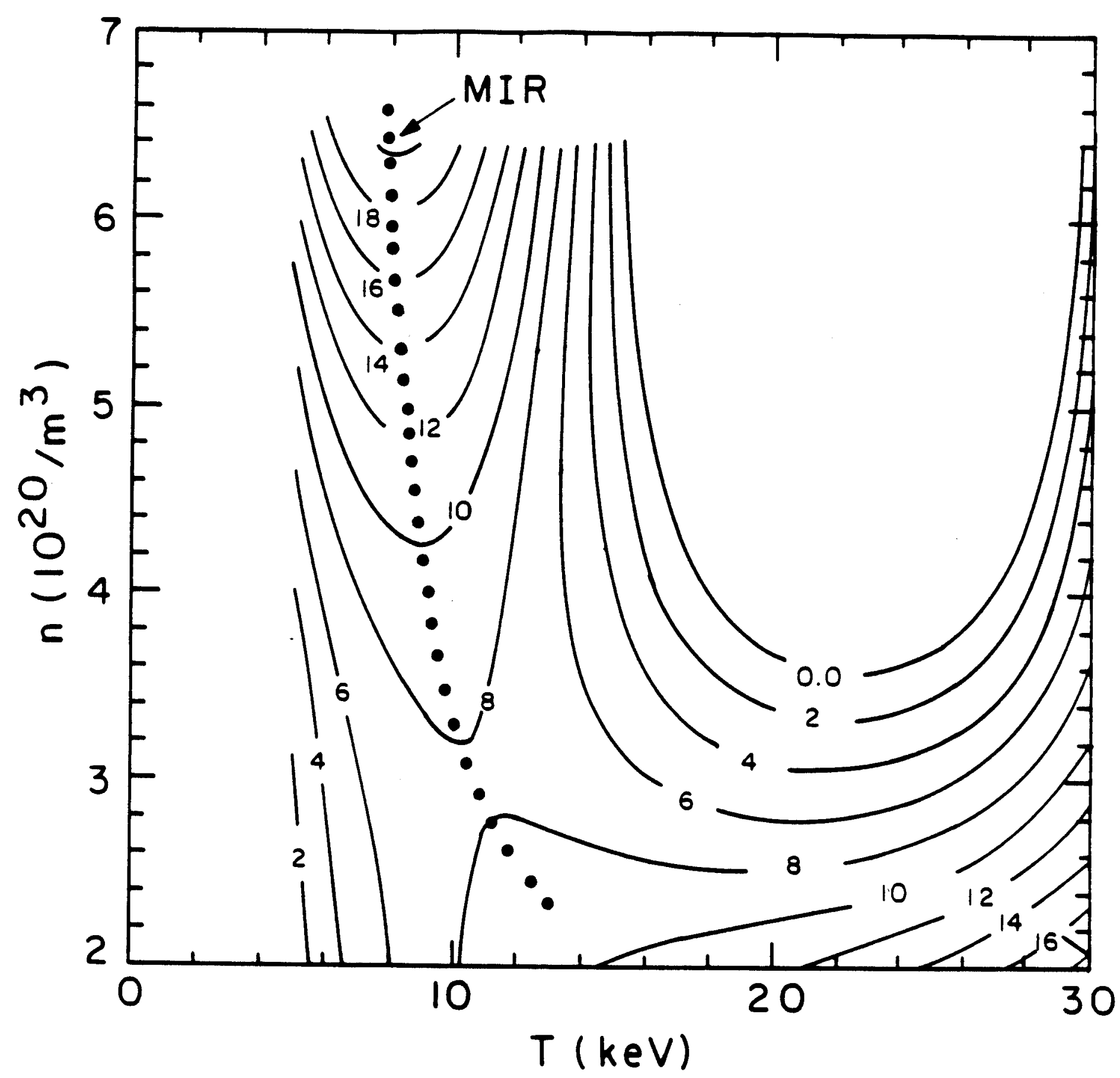

Figure 3: General plasma operating contours of auxiliary power showing the marginal ignition ridge (MIR) contour (dotted line). 
Table 1: CIT Parameters

\begin{tabular}{|ll|r|}
\hline \hline \multicolumn{2}{|c|}{ Parameter } & Value \\
\hline Major Radius & $\mathrm{R}(\mathrm{m})$ & 2.1 \\
Minor Radius & $\mathrm{a}(\mathrm{m})$ & 0.64 \\
Aspect Ratio & $\mathrm{A}$ & 3.28 \\
Elongation & $\kappa$ & 2.0 \\
Toroidal Field & $\mathrm{B}(\mathrm{T})$ & 11.0 \\
Plasma Current & $\mathrm{I}(\mathrm{MA})$ & 11.0 \\
Pulse Length & (sec.) & 5.0 \\
Effective Charge & $Z_{\text {eff }}$ & 1.5 \\
Maximum Auxiliary Power & $P_{a}(\mathrm{MW})$ & 30 \\
\hline \hline
\end{tabular}

\section{Tokamak thermal instability and the need for burn control}

Thermal runaway occurs in a tokamak once the plasma temperature exceeds a certain value. Fig. 1 shows the rate of change of temperature, $\dot{T}$, as a function of temperature, $T$. As is schematically shown in Fig. 2 auxiliary power $P_{I}$ is required in order for the plasma temperature to enter into the ignition regime. If the temperature $T_{I}$ is reached and the auxiliary power is not reduced the plasma temperature continues to increase since $\dot{T}>0$ in this region. It is this temperature increase that we shall call thermal runaway.

In order to distinguish the regions of stable and unstable equilibria we will examine Fig. 4 which shows the $n-T$ operating space of CIT, whose parameters are given in table 1 , under Goldston $\mathrm{H}$-mode scaling. By expanding the energy balance Eq. 24 about the equilibria shown in Fig. 4 the linear growth rate $\gamma\left(\mathrm{sec}^{-1}\right)$ is calculated and its values are shown in Fig. 5 as linear growth rate contours. A positive sign for $\gamma$ indicates the unstable region, while a negative sign implies stable equilibria. Note that there are two regions of stable equilibria. One at low temperatures and the other at high 


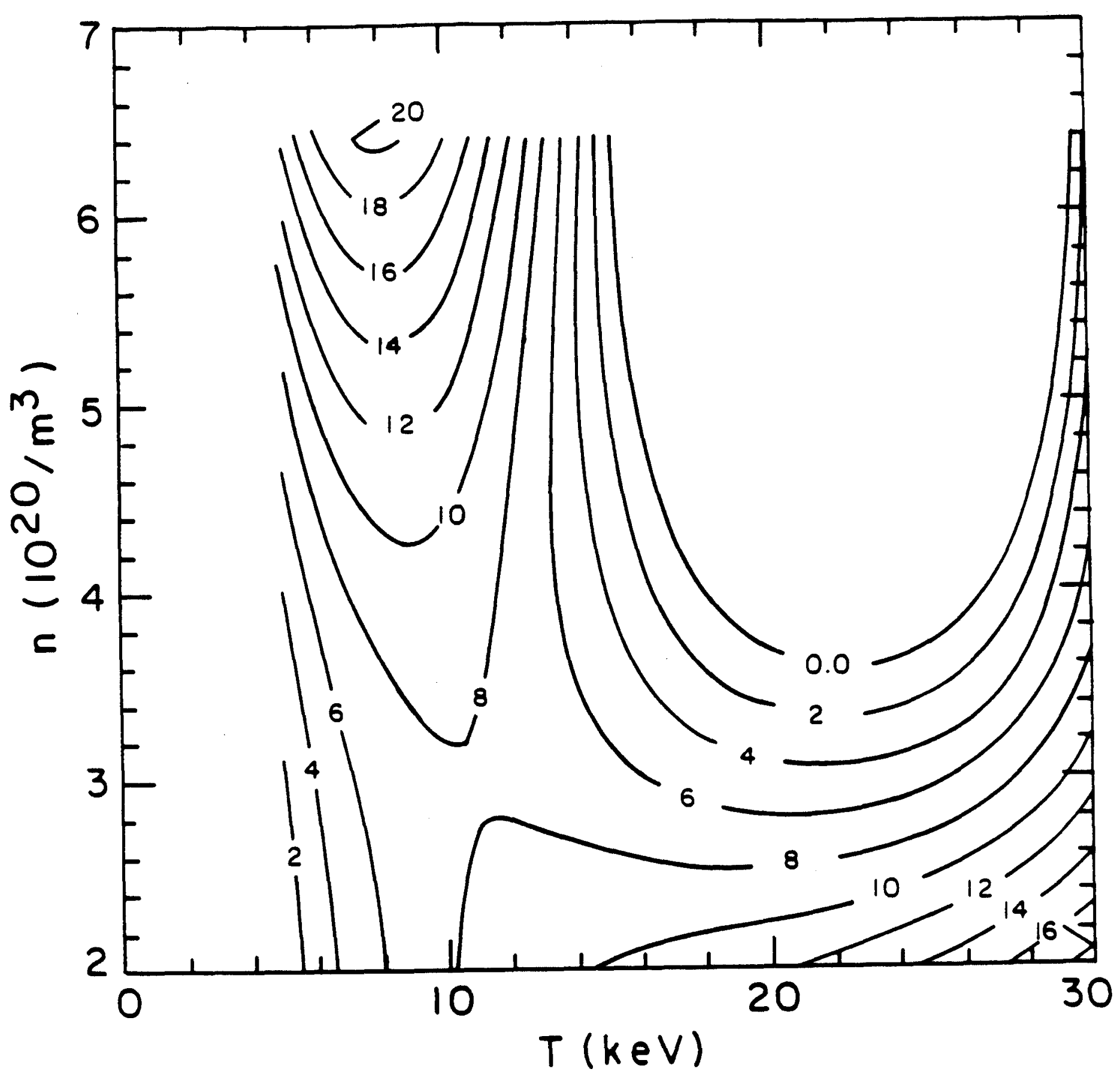

Figure 4: CIT plasma operating contours of auxiliary power for Goldston $\mathrm{H}$-mode scaling 


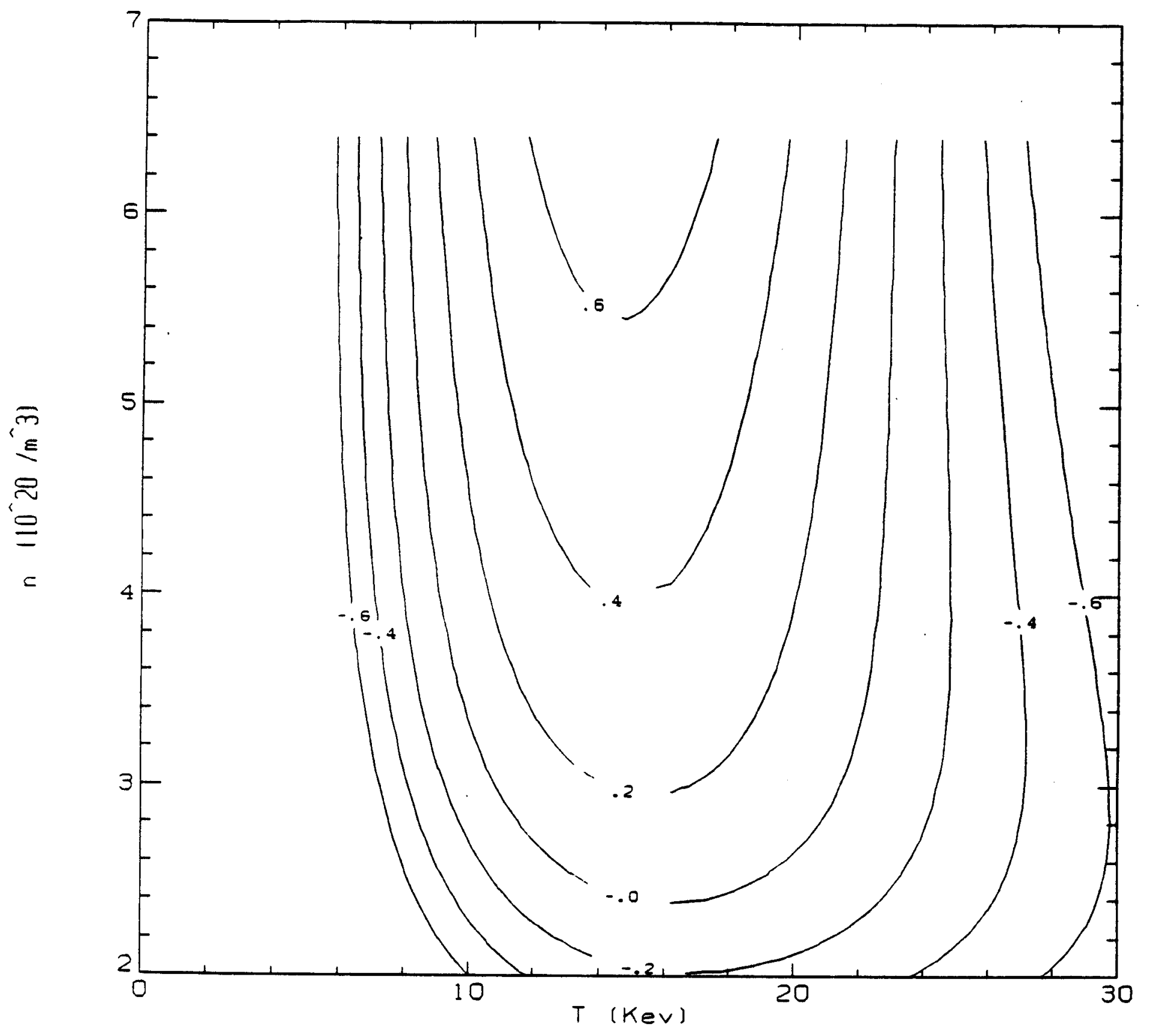

Figure 5: n-T plot with contours of linear growth rates $(\gamma)$ of plasma temperature corresponding to Fig. 4 
temperatures. The low temperature stable equilibria occur at $\sim 5-10 \mathrm{keV}$ depending on the amount of auxiliary power supplied to the plasma. Present day tokamaks operate in this regime and thus all the existing confinement data correspond to this low temperature region of operating space. However, these temperatures are too low for operating an ignition experiment.

In the absence of any physics or technological limits the high temperature stable equilibria could be ideal operating points for an ignited fusion machine since large amounts of fusion power can be produced. However, fundamental limits, such as the Troyon $\beta$ limit and the wall loading limit, exist which can not be violated in a fusion experiment. In Fig. 6 the CIT equilibria corresponding to zero auxiliary power are shown under Neo-Alcator, Goldston-H, and Kaye-Goldston-H scalings. Note that the location of the high temperature equilibria (dashed lines) can vary between 20 and $80 \mathrm{keV}$ depending on the type of confinement assumed. In general, an ignited plasma will evolve to one of these high temperature equilibria. Also on Fig. 6 typical contours for the $\beta$ limit and the wall loading limit of $3 \mathrm{MW} / \mathrm{m}^{2}$ are shown. These limits are violated for some of these equilibria. It is plausible to speculate that the expected uncertainty in the location of these high temperature equilibria will be as large as the variation introduced with present day scalings. During the initial stages of a first generation ignition experiment it is prudent that operation at the high temperature equilibria is not relied upon.

In summary, operation at either of the naturally stable equilibria is not desirable for a first generation ignited tokamak such as CIT. This is because the low temperature equilibria produce insufficient fusion reactions while the high temperature equilibria are unreliable and result in violations of important limits.

Therefore, an ignited tokamak will have to operate in a regime in which the physics and technological limits are not violated. However, these equilibria are unstable requiring some form of feedback control. Since an ignition experiment will have to operate in a regime not previously investigated the existence and the location of these unstable equilibria will be subject to speculation prior to beginning of operation. In the next section the issues related to the investigations of the operating space are addressed. 


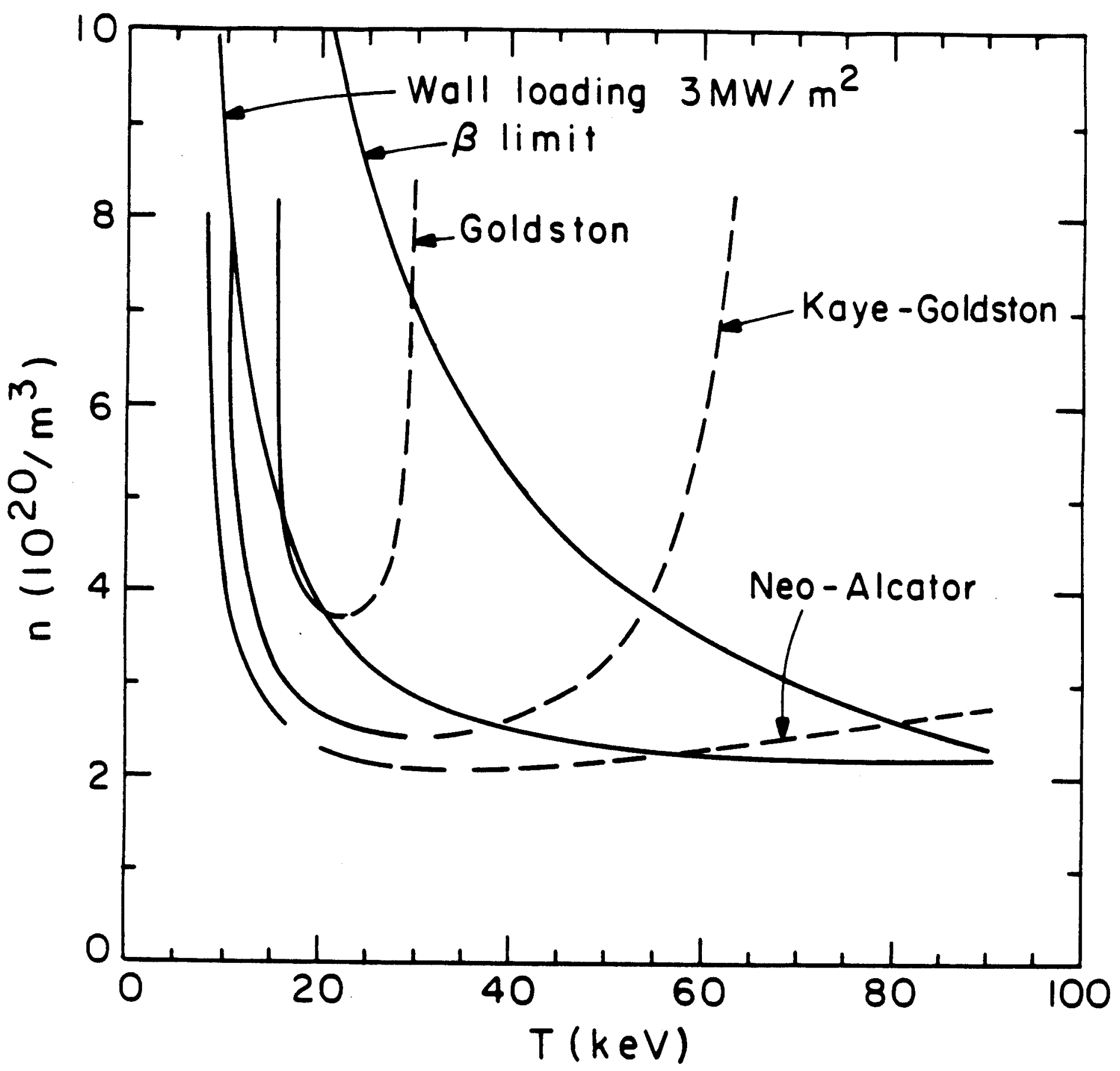

Figure 6: Iocation of the high temperature stable equilibria corresponding to zero auxiliary power under various confinement scalings. 


\section{Mapping the Tokamak Operating Space}

A first generation ignited experiment has two primary goals. First it must be capable of crossing the marginal ignition ridge, and second it must operate at a high enough temperature for performing the necessary studies of an alpha power dominated plasma. The first step towards the achievement of these goals is the determination of the existence of a regime inside which an ignited plasma can operate.

Due to the uncertainty associated with the predictions of existing confinement scalings the characteristics of the operating space in an ignited tokamak are not accurately known. For example, in a steady state POP-CON plot with fixed auxiliary power contours the precise locations of the Cordey pass, the marginal ignition ridge, and the zero auxiliary power contour are unknown. Therefore, determining the characteristics of the operating space, (mapping the operating space) will be the first goal of the ignition experiment. In this section we investigate various methods by which the operating space can be mapped.

During the mapping stage of an ignition experiment the most important goal is to determine the existence and location of the marginal ignition ridge. If such region exists the plasma is capable of igniting according to the definition given in section 4 , i.e. the alpha power will dominate other terms in the energy balance equation. Knowing the location of this ridge is important since it separates the low temperature stable region from the high temperature unstable region. A successful experiment must achieve some form of "stabilized" operation in the high temperature region.

Auxiliary power, whose primary objective is to assist the plasma in crossing the marginal ignition ridge, may be used for mapping the tokamak operating space both in the low temperature stable regime and in the high temperature ignition regime. The primary motivation for using auxiliary power is that it is capable of creating steady state equilibria at any location, 
provided that sufficient power is available. For a fixed plasma density a certain auxiliary power $P_{I}$ is required to enter the ignition regime. For $P_{a}<P_{I}$ an equilibrium point if reached to the left of the MIR. If $P_{a}>P_{I}$ the plasma temperature will continue to increase, resulting in a thermal runaway, unless the power is appropriately reduced. The procedure by which the auxiliary power is changed is outlined in section 9 in the burn control part of the report.

In an experiment, the location of the marginal ignition ridge can be determined by either (1) measuring the energy confinement time and extrapolating (2) measuring $Q$ (Fusion power / Auxiliary power) as a function of temperature, (3) looking for an inflection point in the temperature versus time plot. In this analysis we assume that acceptable reproducibility exists from shot to shot, and thus we assume that once the behavior at a certain point is known it will remain the same as long as the operating conditions do not change.

The energy confinement time may be measured during the temperature evolution or more easily during a temperature flat-top. By using auxiliary power, as outlined above, temperature equilibria can be created. By measuring the peak temperature and density as well as their profile shapes during the flat-top the energy confinement time $\tau_{E}$ is essentially deduced from the volume average of the equation

$$
P_{a}=\frac{n T}{\tau_{E}}+P_{b}-\frac{P_{\text {fusion }}}{5}-P_{\Omega}
$$

The fusion power may be deduced from the density and temperature measurements or directly from appropriately calibrated neutron detectors. The amount of auxiliary power absorbed by the plasma is estimated by modeling the energy transfer mechanism of the particular form of auxiliary power used.

Once the fusion power produced by the plasma $P_{f}$ and the auxiliary power $P_{A}$ absorbed by the plasma are known the value of $Q=P_{f} / P_{a}$ is estimated. 
For a given density the value of $Q$ at the marginal ignition ridge is found by simultaneously solving the equations

$$
\begin{aligned}
\frac{d T}{d t} & =0 \\
\frac{d P_{a}}{d T} & =0
\end{aligned}
$$

In Fig. 7 the value of $Q$ at the marginal ignition ridge of CIT is plotted as a function of plasma density $n$ under various confinement scalings. Note that $Q$ ranges from 4 to 6 as the density varies from its value at the Cordey pass to the Murakami limit. It is therefore obvious that a $Q$ of at least 5 must be achieved before any claim for an ignited plasma can be made. Any other situation for which the operating space does not have a marginal ignition ridge in the regime of interest is incapable of producing $Q=5$. Therefore, once $Q=5$ is detected the implication is that the plasma is capable of igniting. The importance of the $Q=5$ contour is also shown in Figs. 8, 9 , and 10 where the marginal ignition ridge (MIR) and the $Q=5$ contour are shown for Neo-Alcator, Kaye-Goldston H-mode, and Goldston H-mode confinement scalings. Note that the $Q=5$ contour almost coincides with the MIR for all the scalings shown.

Another indicator for the existence and location of the MIR is the appearance of an inflection point in the trace of temperature versus time. Fig. 11 shows a plot of temperature $v s$ time for a shot with the capability of crossing into the ignition regime. Note that at a certain time the temperature evolution shows an inflection point. The temperature at which the inflection point occurs lies in the MIR and thus the observation of an inflection point signifies that the plasma has entered the ignition regime.

However detecting the inflection point will be difficult in an experiment. The existence of experimental errors as well as the effect of large amounts 


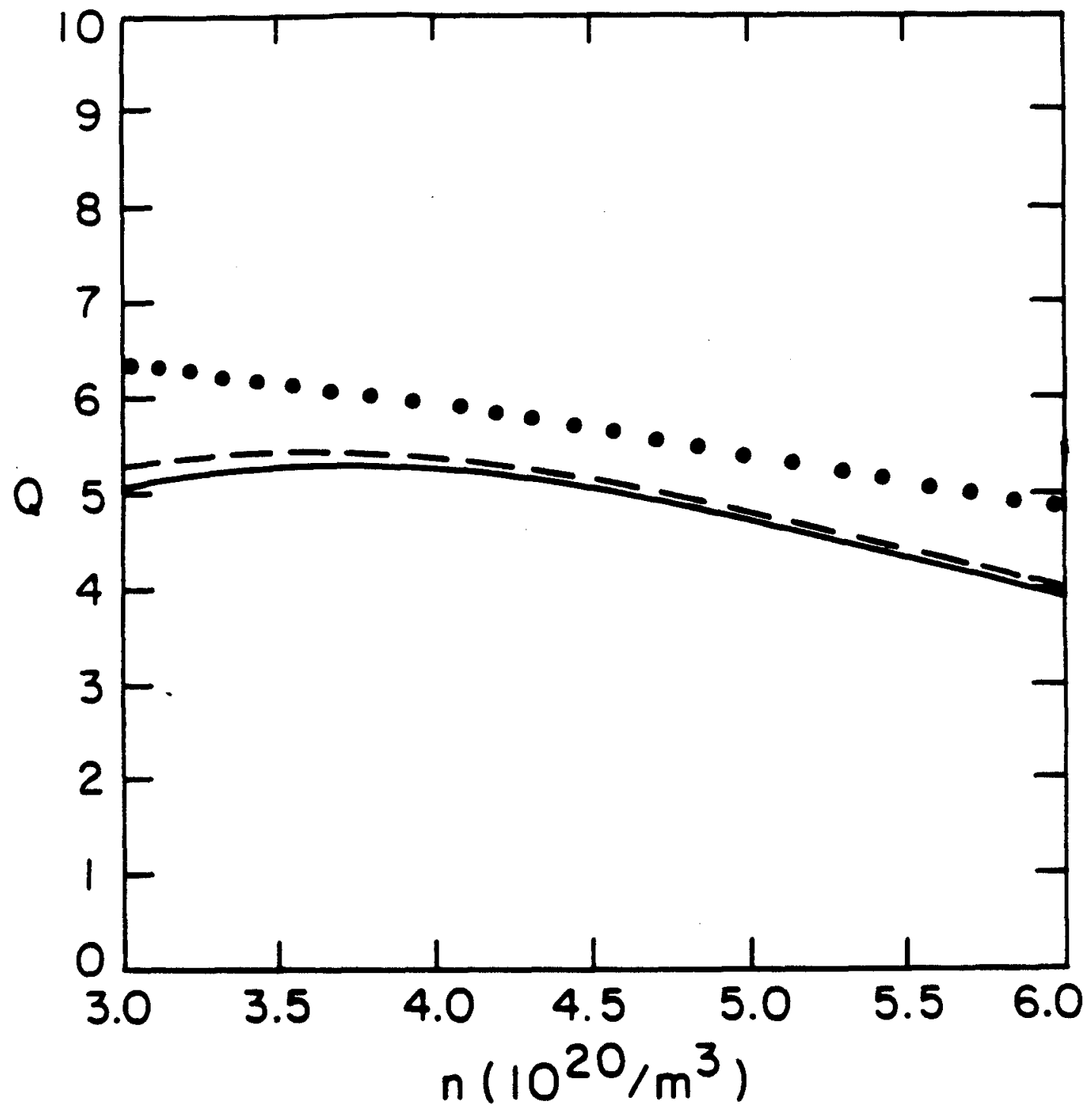

Figure 7: $Q$ at the marginal ignition ridge (MIR) as a function of plasma density for Neo-Alcator (solid line), Goldston (dotted line), and Kaye-Goldston (dashed line) confinement scalings 


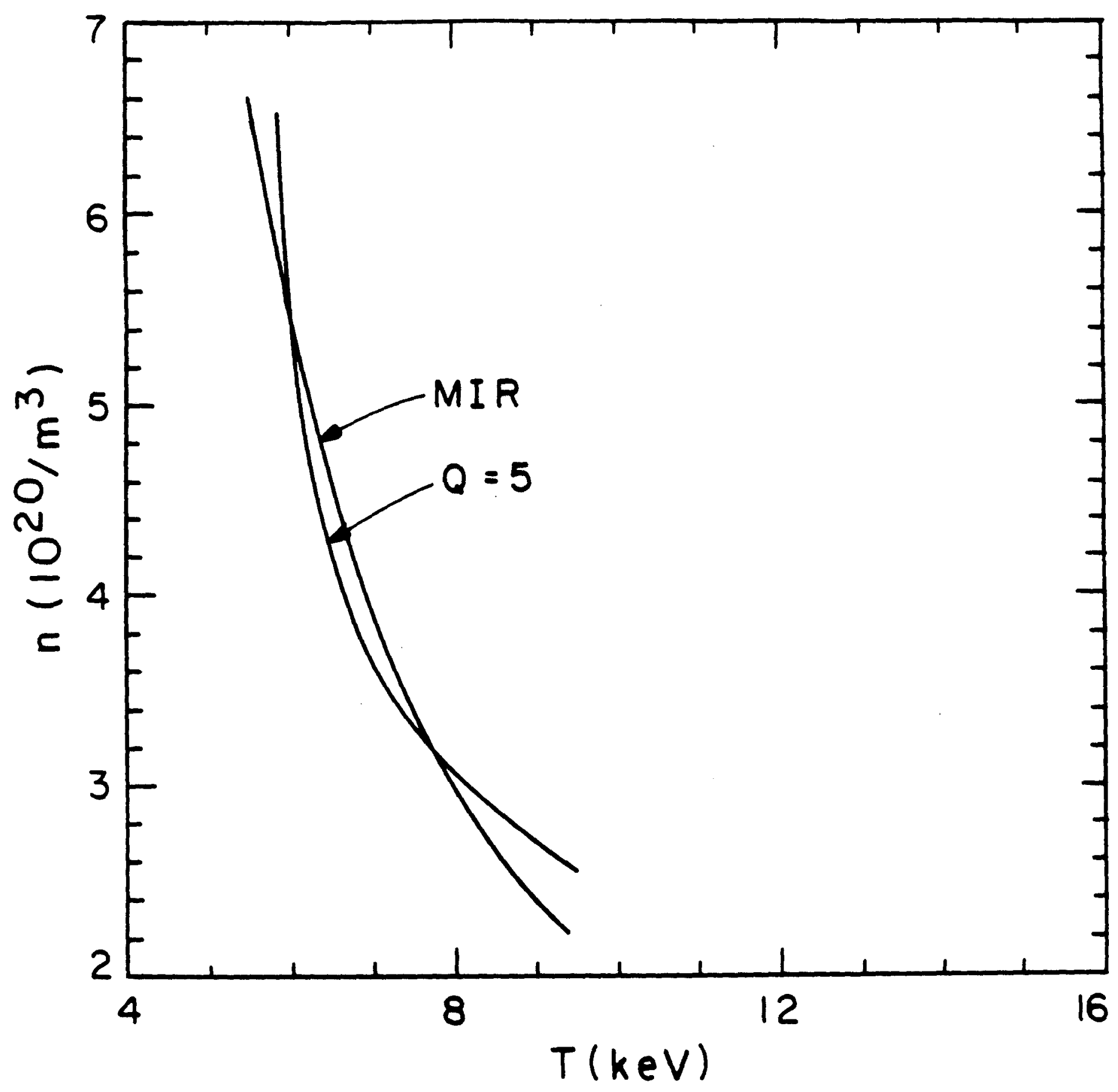

Figure 8: The marginal ignition ridge (MIR) and the $Q=5$ contour are shown for CIT under Neo-Alcator scaling 


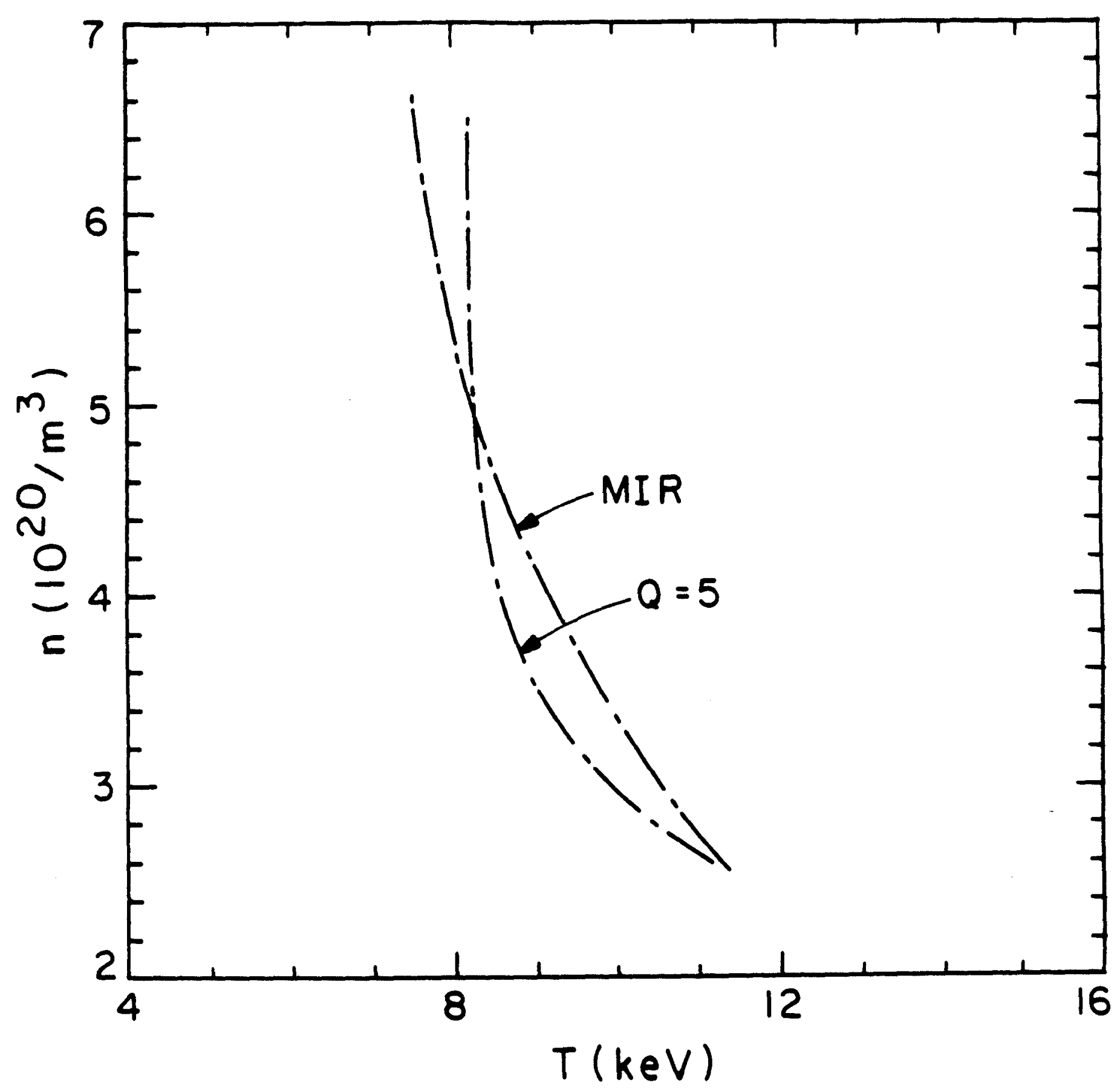

Figure 9: The marginal ignition ridge (MIR) and the $Q=5$ contour are shown for CIT under Goldston H-mode scaling 


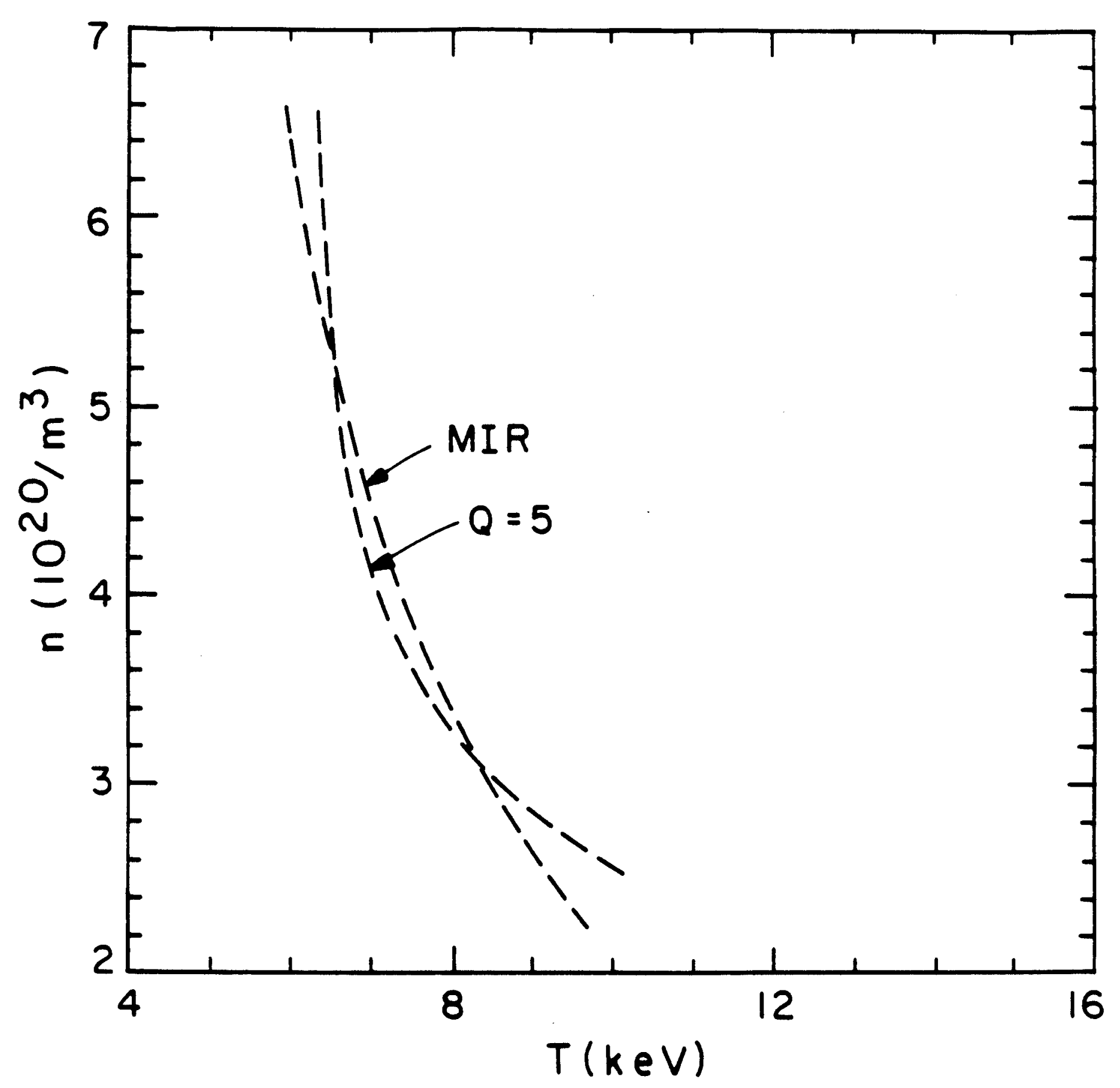

Figure 10: The marginal ignition rirge (MIR) and the $Q=5$ contour are shown for CIT under Kaye-Goldston H-mode scaling 


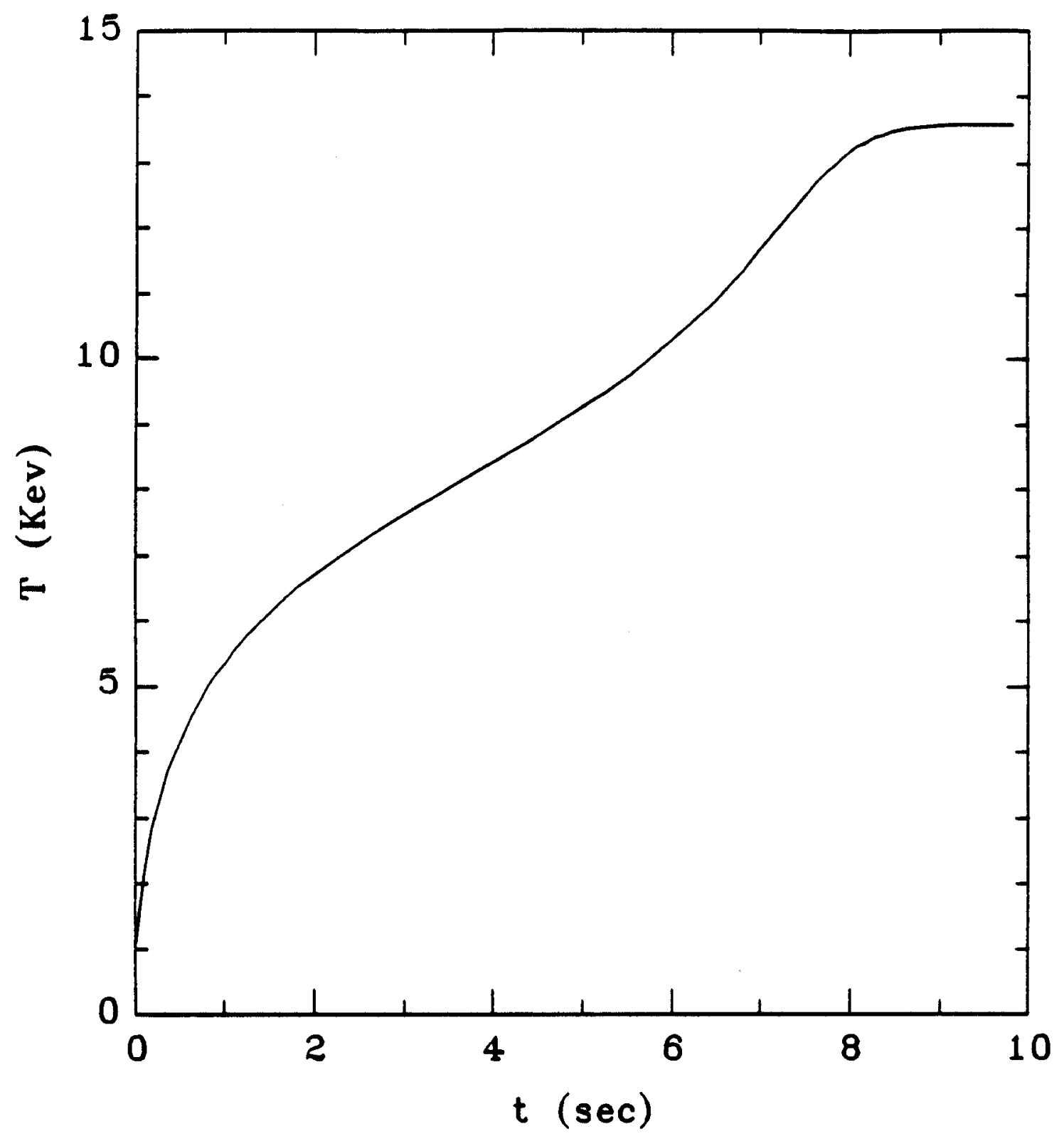

Figure 11: Temperature evolution of an ignited CIT with "small" amount of auxiliary power. 


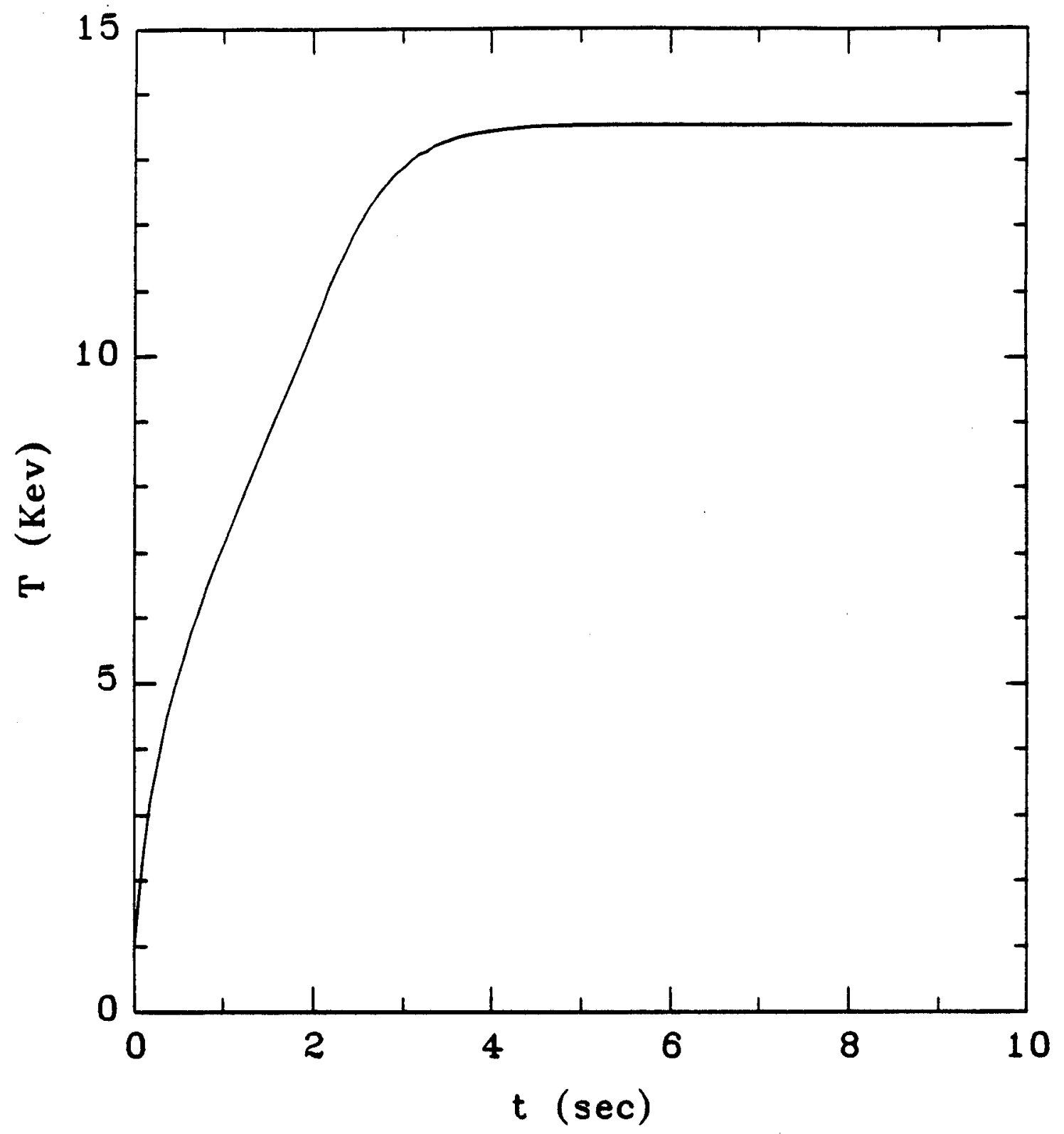

Figure 12: Temperature evolution of an ignited CIT with "large" amount of auxiliary power. 
of auxiliary power make the detection of an inflection point very uncertain. For example note the shapes of the two curves shown in Figs. 11, and 12. Even thought the same final temperature has been reached the curve with the small amount of auxiliary power (Fig. 11) has a clear inflection point while the location of the inflection point in the curve corresponding to the large amount of power (Fig. 12) is very uncertain. For these reasons this method does not appear to be very helpful during the mapping process of the tokamak operating space. 


\section{Burn Control: General Remarks}

Once the existence and the location of the MIR is determined operation in the high temperature alpha dominated regime is possible. Here we assume that the operating space resembles the one shown in Fig. 4.

We begin the analysis by briefly reviewing the various burn control mechanisms suggested to date. Next we identify the most important issues that must be addressed by a control systern. Consideration of the major advantages and disadvantages of each control system leads to the conclusion that auxiliary power modulation is the most desirable method for a first generation ignited tokamak. A detailed discussion of auxiliary power control as well as a complete control model will then be presented. Finally, specific examples of ignited and controlled plasmas are presented.

\section{Burn Control Methods.}

Over the years researchers have suggested a variety of methods for providing temperature control to a burning plasma. In this section we review the most widely studied methods by briefly explaining the physical principle and outlining the major advantages and disadvantages of each. The primary motivation for the selection of a particular burn control method is reliability, both technological and scientific. While a number of methods may actually be tested in an ignited fusion experiment, it is clearly important that the baseline design must be based on the ones with the most reliability in order to insure overall credibility. The various control mechanisms may be classified as passive or active depending on whether the modifications to the energy transport in the plasma is achieved by the plasma itself (passive) or by actively altering the plasma operating conditions.

\subsection{Passive Methods}

\subsubsection{Soft $\beta$ Limit Control}

It has been suggested $[6,7,8]$ that the plasma may automatically provide itself with burn control by means of a soft $\beta$ limit. In particular, as the plasma 
undergoes a thermal runaway, it will eventually exceed the Troyon $\beta$ limit. To the extent that this is a "soft" limit, the consequence is a rapid deterioration of confinement time, resulting in automatic burn control. Economically and technologically such a method is highly desirable. However, the existence and behavior of a soft $\beta$ limit is not well established. Thus, the method does not have high scientific reliability. Furthermore, if the $\beta$ limit is hard rather than soft, the result is a major disruption, leading to potentially serious damage to the first wall. Furthermore, the $\beta$ limit must also be less than the wall loading limit, particularly in a reactor.

\subsubsection{Field Ripple Control}

Another method $[9,10,11,12,13]$ of natural burn control relies on the prediction, from neo-classical transport theory, that toroidal field ripple can significantly enhance ion heat transport. To achieve control, the magnetic field is designed so that the ripple increases strongly with major radius near the outside of the torus. Thus, as the plasma heats during a thermal runaway, its Shafranov shift increases, moving the plasma into a region of strong ripple. This provides automatic (natural) burn control. Again the primary advantages of such a method are its economic and technological attractiveness. The problems with ripple control are as follows. First, the enhanced transport resulting from field ripple has not been reliably verified experimentally. Second, if the plasma ignites in an optimized region of minimum field ripple, it is not possible to stabilize against negative thermal excursions. Finally, if one needs to stabilize relatively large positive temperature deviations, the field ripple must be actively increased over a short time scale. However, due to the long time scale associated with magnetic field diffusion, this may be impossible to accomplish.

\subsection{Active Methods}

\subsubsection{Vary $B_{0}$.}

If the toroidal magnetic field $B_{0}$ is varied above and below the value which gives the desired equilibrium temperature, then for essentially all confinement 
scalings the performance parameter $n \tau$ varies accordingly. This in principle should be an effective way to stabilize both positive and negative temperature excursions. The major difficulty with this procedure is that the magnetic diffusion time $\tau_{D} \approx \mu_{0} a^{2} / \eta$, where $a$ and $\eta$ are respectively the radius and resistivity of the plasma, is very long, thus prohibiting effective stabilization of fast temperature excursions. Also, such a control system requires that the value of the steady state toroidal magnetic field be below the design limit in order to insure that negative temperature deviations can be stabilized. Furthermore, the power requirements for varying the magnetic field may be prohibitively large.

\subsubsection{Adiabatic Expansion / Compression}

By varying the vertical field during a thermal runaway, the plasma can expand (or compress) adiabaticlly $[14,15,16,17]$. For an expansion, the resulting state has lower $n$, lower $T$, larger $R$ and larger $a$. In most circumstances the strong $n, T$ dependence of the alpha particle heating dominates. This cools the plasma, thereby providing a mechanism for burn control. The difficulties with this method involve power requirements on the vertical field circuit, and the need for a somewhat oversized vacuum chamber to allow room for expansion and contraction. The problem is more serious in configurations with divertors, since there is in general only very limited flexibility in the allowable motion of the $X$ points of the separatrix.

\subsubsection{Vary the Plasma Density $n_{0}$}

If the density can be varied above and below its thermal equilibrium value, the resulting change in alpha particle power should be adequate to provide burn control. There are several important difficulties with this method. Since the density diffusion time scale is typically three to five times longer than 
the energy confinement time, a combination of gas puffing and particle diffusion does not have adequate response time to stabilize the thermal runaway. Part of the difficulty can be overcome by injecting hydrogen pellets into the plasma. The increased mass cools the plasma but does not produce any additional alpha particles. This process takes place on a very short time scale, more than adequate for burn control. Thus, pellet injection can effectively stabilize positive thermal excursions. Negative temperature excursions are more difficult to control. Injecting pellets of $\mathrm{D}$ or $\mathrm{T}$ should in principle increase the plasma reactivity. However, once a pellet is injected, the immediate plasma response is a drop in $\mathrm{T}$ and an increase in $\mathrm{n}$, further amplifying the initial negative temperature excursion. Whether or not the temperature can recover quickly enough to reheat the plasma is uncertain.

\subsubsection{Vary the Auxiliary Power $P_{a}$}

Since a certain auxiliary power is required for ignition, lowering $P_{a}$ suffciently fast during the thermal runaway $[18,19,20]$ can cause a net loss in plasma energy; the decrease in plasma energy due to lower $P_{a}$ exceeds the increase due to the thermal runaway in $P_{\alpha}$. This procedure can stabilize a positive thermal excursion. Conversely, rapidly increasing $P_{a}$ during a negative temperature excursion, also produces stability. Auxiliary heating in the form of RF power can be varied in a relatively continuous manner on a virtually instantaneous time scale. In this regard, varying $P_{a}$ is an effective way to achieve burn control. The primary disadvantage is that in order to stabilize both positive and negative temperature excursions, the value of $P_{a}$ must be non-zero at the point of thermal equilibrium. Therefore, the overall efficiency of the reactor, defined by $Q \equiv$ Fusion power $\left(P_{f}\right) /$ Auxiliary power $\left(P_{a}\right)$, can be unacceptably low if the maximum temperature excursion to be stabilized is too large (requiring a large equilibrium $P_{a}$ ).

From the discussion above, it is apparent that there are a wide variety of possible burn control methods. Each has advantages and disadvantages, and 
none is sufficiently simple and well tested experimentally to assure success. Passive burn control methods such as soft the $\beta$ limit control or field ripple are more desirable from an economic point of view. It is therefore important that future fusion experiments carry out studies aimed at investigating the validity of such methods. However, in judiciously designing near term experiments such as CIT and ITER the primary burn control method must utilize the most reliable method available.

In this paper we recommend that burn control by means of varying $P_{a}$ is the best choice for the first generation ignition experiments. Significant auxiliary power already exists for ignition, so no additional major hardware is required. Varying $P_{a}$ provides stability against both positive and negative temperature excursions. The main drawback is that in steady state the plasma must operate sub-ignited requiring a finite non-zero value of $P_{a}$. However, we show in the analysis that follows, that the $Q$ of the system (fusion power over the auxiliary power) remains quite high for reasonable values of the thermal excursion $\Delta T$ that must be stabilized. For these reasons, the remainder of the analysis is focussed on burn control by means of varying $P_{a}$. 


\section{Burn Control Issues}

The goal of burn control is to provide a stable thermal equilibrium in the regime of alpha dominated ignited operation. In general, any viable burn control study requires consideration of the following issues: determination of the equilibrium "operating" temperature, stability analysis about the operating temperature, calculation of the time scales involved in the problem, and evaluation of the various diagnostic and engineering issues. These issues are described as follows:

\subsection{Thermal Equilibrium}

In order to achieve steady state operation the power balance must by definition satisfy $\dot{T}=0$. Furthermore, for fully ignited operation power balance must be satisfied for $P_{a}=0$. As indicated in section 5 there exists one temperature, labeled $T_{B}$, for which both $\dot{T}=0$ and $P_{a}=0$ in the regime of interest. From Fig. 2 we see that the temperature $T_{B}$ is accessible once the plasma crosses the MIR. Also, note from Fig. 2 that if one wishes to operate at a lower intermediate temperature $T_{I}<T<T_{B}$, then a finite $P_{a}$ is required for thermal equilibrium.

\subsection{Stable Operation}

Once in thermal equilibrium, the plasma must lie in a region of stable operation. Specifically, if the plasma experiences a small perturbation in temperature, the shape of the $\dot{T}$ vs $T$ curve must be such that the plasma returns to its equilibrium operating point. In a linear analysis this requires

$$
\left.\frac{\partial \dot{T}}{\partial T_{S}}\right|_{T_{s}}<0
$$

where $T_{S}$ is the desired operating temperature. 
Observe that as the auxiliary power is gradually reduced to zero the operating temperature approaches $T_{B}$. At $T_{B}$ Eq. 35 is not satisfied and thus $T_{B}$ is an unstable equilibrium. Providing a realistic method for stabilizing operation about $T \approx T_{B}$ is the primary goal of burn control studies.

\subsection{Time Scales}

A critical feature of any burn control system is its response time. In the case of an active control scheme, there may be a substantial delay between the time that the temperature of the plasma is detected and the time that the system responds by altering the plasma temperature for stability. For effective stabilization against temperature excursions, the burn control system must be able to respond on a time scale shorter than the thermal runaway time $\tau_{R}$. In general, the e-folding thermal runaway time $\tau_{R}$ is given by

$$
\tau_{R}=\frac{3 n}{\left.\frac{d}{d T}\left(P_{\alpha}+P_{n}+P_{a}-P_{l}-P_{b}\right)\right|_{T_{S}}}=\left(\frac{\partial \dot{T}}{\partial T_{S}}\right)^{-1}
$$

Depending on the type of confinement scaling used $\tau_{R}$ varies between 0.5 and 2.0 seconds in the regime of interest.

The delay time associated with the deposition of power into the plasma is an important consideration in a control system. The alpha particles deliver most of their energy to the electrons in an e-folding time given by

$$
\tau_{\alpha e}=\begin{aligned}
& 0.099 T^{3 / 2} \\
& \ln \Lambda n
\end{aligned} \quad(\mathrm{sec})
$$

Similar estimates can be made for the time delay in the energy transfer between the auxiliary power mechanism and the plasma particles. The time delay associated with the feedback system must also be included as well as detection delays. 


\subsection{Diagnostic and Engineering Issues}

Burn control methods based on active stabilization of temperature excursions depend on monitoring the plasma parameters. Therefore, the overall response of the feedback control system is determined by both the inaccuracies of the diagnostics and the finite delays inherent in the system. Deviations from the stable equilibrium temperature cause changes in the fusion power production that introduce damaging cyclic heat and neutron fluxes to the first wall and supportive structures. Therefore, it is important to keep temperature excursions to a minimum. This requires accurate temperature measurements and fast response of the feedback system.

A successful burn control system must satisfy each of the conceptual requirements described above as well as a large number of detailed practical problems. 


\section{Auxiliary Power Control: General Re- marks}

Burn control with auxiliary power modulation is obtained by varying the input power as a function of plasma parameters such as temperature or fusion power (neutron flux). At the beginning of the discharge the maximum amount of auxiliary power is supplied to the plasma. Once the temperature crosses the MIR the plasma enters into the unstable ignition regime. Once in this regime the auxiliary power will be appropriately reduced in order to create an equilibrium at the desired temperature. Quantitatively the auxiliary power term in the energy balance, (Eq. 24) is varied according to the proportional feedback control law:

$$
P_{a}(T)=\left\{\begin{array}{cl}
P_{I}+\Delta P & T<T_{1} \\
\left(P_{I}+\Delta P\right)\left[1-\left(\begin{array}{c}
T-T_{1} \\
T_{2}-T_{1}
\end{array}\right)^{\lambda}\right] & T_{1}<T<T_{2} \\
0 & T>T_{2}
\end{array}\right.
$$

where $P_{I}$ is the minimum auxiliary power required to cross the MIR, $\Delta P$ is excess power above this value required for a finite time evolution to the desired operating point, $T_{1}$ is a temperature $\left(\sim T_{I}\right)$ above which the auxiliary power is gradually reduced, and $T_{2}$ is a temperature $\left(\sim T_{B}\right)$ between $T_{1}$ and the burn temperature $T_{B}$ at which $P_{a}=0$. The exponent $\lambda$ dictates the shape of the $P_{a}$ versus $T$ curve.

By changing the parameters in Eq. 38 a steady state operating temperature can be created somewhere between $T_{1}$ and $T_{B}$. Figure 13 illustrates (a) the original system, (b) the form of the applied auxiliary power, and (c) the final system with steady state operating temperature $T_{S}$.

Initially, auxiliary power equal to $P_{I}+\Delta P$ is supplied to the plasma. By doing so the plasma temperature increases and eventually the MIR temperature $T_{I}$ is reached. The temperature continues to increase until the value $T_{1}$ 

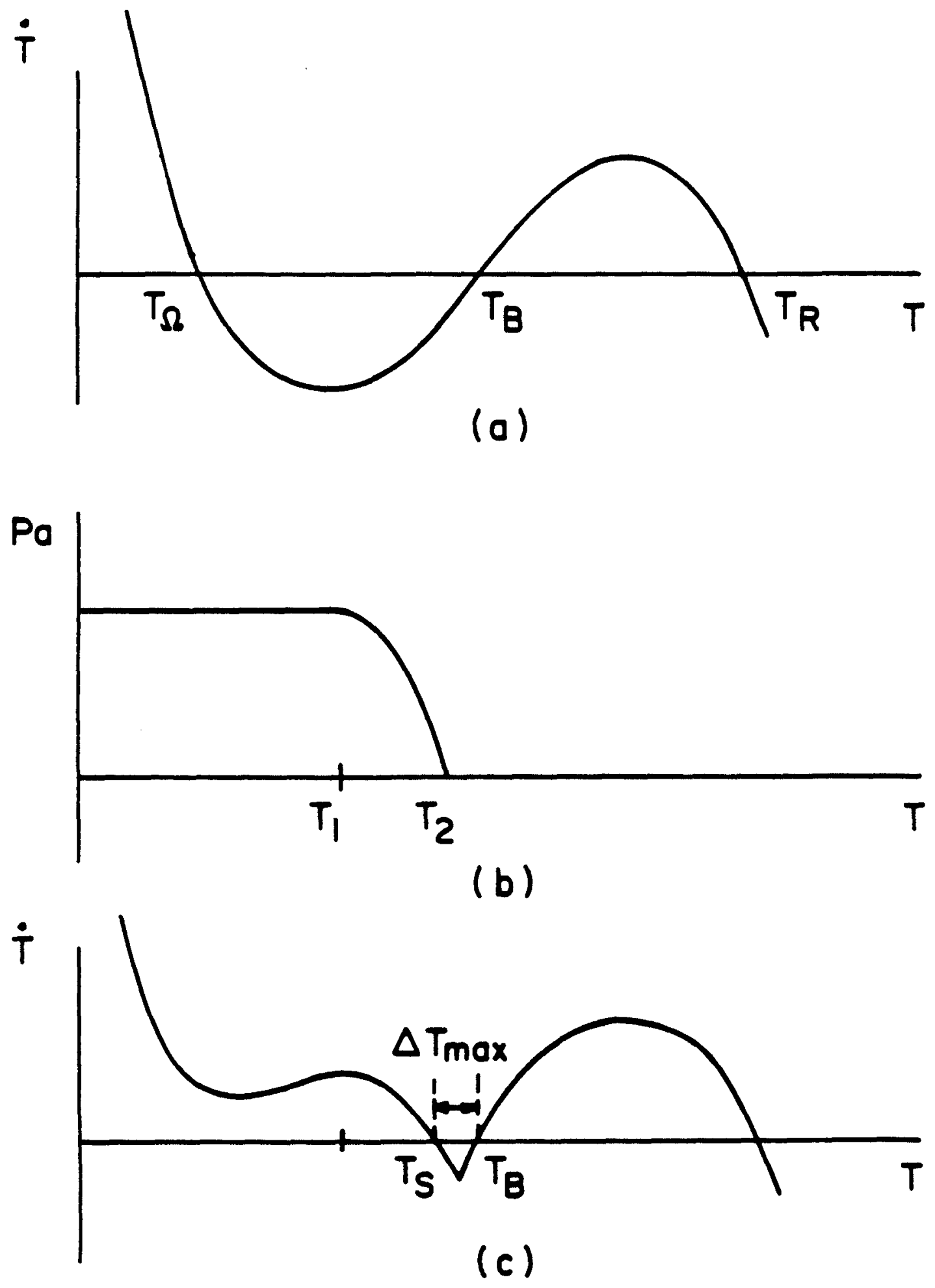

Figure 13: Schematic representation of an original system (a), the form of the auxiliary power (b), and the final system indicating the steady state operating temperature $T_{S}$ 
is reached. The auxiliary power is then decreased according to the functional form given by Eq. 38. In the absence of plasma disturbances the temperature continues to increase since $\dot{T}>0$ until the operating temperature $T_{S}$ is reached. Note that operation at the temperature $T_{S}$ requires a non-zero steady state auxiliary power $P_{S}$. From an energy efficiency perspective it is important that $P_{S}$ be as small as possible.

Stabilization against temperature excursions away from the operating temperature $T_{S}$ is obtained by altering the amount of auxiliary power supplied to the plasma. During a positive temperature excursion the auxiliary power must be decreased from the steady state value $P_{S}$. This results in an overall energy loss from the plasma and thus a decrease in temperature. If the temperature decreases from the operating point, $T_{S}$, the input power is increased. As a result, the overall plasina energy and consequently the plasma temperature increases returning operation to $T_{S}$. Therefore, the auxiliary power should be viewed as a source of both power enhancement and power loss. Power enhancement occurs to the left of the operating temperature $T_{S}$, and power loss occurs to the right of $T_{S}$. This type of control about the operating temperature $T_{S}$ breaks down for positive temperature excursions $\Delta T$ above a certain magnitude. In an ideal system (i.e. one without time delays and with instantaneous feedback response) the maximum temperature deviation that can be stabilized is $\Delta T_{\max }=T_{B}-T_{S}$ (see Fig. 13). Similarly in an ideal system all negative excursions are stabilized.

\section{The Complete Model}

In addition to the power balance Eq. 24, a complete burn control model must include the effects of the time delays discussed in section 8 , the form of the feedback law given in section 9 as well as the density evolution. 


\subsection{Time Delays}

In our model we incorporate the time delay associated with the energy transfer between the fusion alpha particles and the plasma ions as well as the time delay associated with the deposition of auxiliary power into the plasma.

In general, the effect at the present time $t$ of a stimulus $S_{1}\left(t^{\prime}\right) d t^{\prime}$ at any past time $t^{\prime}$ is given by

$$
S_{2}(t)=\int_{0}^{t} \mathcal{R}\left(t-t^{\prime}\right) S_{1}\left(t^{\prime}\right) d t^{\prime}
$$

where we have assumed that the effect is proportional to the stimulus with proportionality constant $\mathcal{R}$ which depends only on the elapsed time $t-t^{\prime}$. Hence it has the form $\mathcal{R}\left(t-t^{\prime}\right)$. Eq. 39 gives the response at the present time $t$ as a weighted superposition over the input at the times $t^{\prime}<t$. The weighting factor $\mathcal{R}\left(t-t^{\prime}\right)$ characterizes the system, and $S_{1}\left(t^{\prime}\right)$ characterizes the past history of the input.

In particular, since we are concerned with the delay time associated with the transfer of energy as particles collide, the delay time or the energy transfer time $\tau$ is given by

$$
\tau=-\frac{E}{d E / d t}
$$

where $E$ is the particle energy. By integrating Eq. 40 we obtain for the rate $R$

$$
\mathcal{R}\left(t_{1}\right)=-\frac{d E\left(t_{1}\right)}{E_{0}} \frac{1}{d t_{1}}=\frac{1}{\tau} \exp \left[-\frac{t_{1}}{\tau}\right]
$$

In Eq. $41 t_{1}=t-t^{\prime}$ and represents the time elapsed since the particle had energy $E_{0}$. By substituting Eq. 41 into Eq. 39 and differentiating with respect to $t$ we obtain

$$
\frac{d E_{2}(t)}{d t}=\frac{1}{\tau}\left[E_{1}(t)-E_{2}(t)\right]
$$


This is now a differential equation giving the effect of the stimulus, in this case the amount of energy transferred into the plasma, at time $t$ subject to the delay time $\tau$ and some appropriate initial condition.

Similarly the time response of the feedback system, including the diagnostics and the auxiliary power delivery system, can be modeled by evaluating the auxiliary power at a delayed temperature $T_{d}$. A simple model for the feedback system is obtained by assuming that the delayed temperature $T_{d}$ relaxes to the plasma temperature with a time constant $\tau_{d}[19]$

$$
\stackrel{d T_{d}}{d t}=\frac{1}{\tau_{d}}\left(T-T_{d}\right)
$$

\subsection{Density Evolution}

As the plasma temperature evolves the plasma density is changing due to fusion processes, fueling, volumetric effects, and particle confinement. In general the evolution of the deuterium $\left(n_{d}\right)$ and tritium $\left(n_{t}\right)$ densities has the form

$$
\begin{aligned}
& \frac{d n_{d}}{d t}=S_{d}-2 f_{d}\left(1-f_{d}\right) \overline{\sigma v} n^{2}-n_{d} \frac{1}{V} \frac{d V}{d t}-\frac{n_{d}}{\tau_{p}} \\
& \frac{d n_{t}}{d t}=S_{t}-2 f_{d}\left(1-f_{d}\right) \overline{\sigma v} n^{2}-n_{t} \frac{1}{V} \frac{d V}{d t}-\frac{n_{t}}{\tau_{p}}
\end{aligned}
$$

where $S_{d}\left(S_{t}\right)$ is the volumetric deuterium (tritium) source rate, $f_{d}$ gives the deuterium fraction in the plasma, $V$ is the plasma volume, and $\tau_{p}$ is the particle confinement time.

By assuming that deuterium and tritium have identical confinement properties the total deuterium-tritium density, $n=n_{d}+n_{t}$ is given by

$$
\frac{d n}{d t}=S-2 f_{d}\left(1-f_{d}\right) \overline{\sigma v} n^{2}-n \frac{1}{V} \frac{d V}{d t}-\frac{n}{\tau_{p}}
$$


where $S=S_{d}+S_{t}$ is the total volumetric source rate. The variation in the deuterium fraction, $f_{d}$, is obtained by differentiating Eq. 5 with respect to time

$$
\frac{d f_{d}}{d t}=\left(f_{s, d}-f_{d}\right) \frac{S}{n}-\left(1-2 f_{d}\right) f_{d}\left(1-f_{d}\right) \overline{\sigma v} n
$$

where $f_{s, d}$ is the fraction of deuterium in the source. By assuming profiles of the form given by Eqs. 8, and 9 the volume average of Eqs. 46, and 47 becomes

$$
\begin{aligned}
\frac{d n}{d t}= & \left(1+\nu_{n}\right)\langle S\rangle-0.02 \frac{1+\nu_{n}}{\nu_{T}} f_{d}\left(1-f_{d}\right) F_{\alpha}(T) n^{2}- \\
& n \frac{1}{V} \frac{d V}{d t}-\frac{n}{\tau_{p}} \\
n \frac{d f_{d}}{d t}= & \left(1+\nu_{n}\right)\left(f_{s, d}-f_{d}\right)\langle S\rangle- \\
& 0.01 \frac{1+\nu_{n}}{\nu_{T}}\left(1-2 f_{d}\right) f_{d}\left(1-f_{d}\right) F_{\alpha}(T)
\end{aligned}
$$

In the above equations the volumetric source rate, $S$, is given in units of $10^{20} / \mathrm{m}^{3} / \mathrm{sec}$, the density in units of $10^{20} / \mathrm{m}^{3}$, and $\tau_{p}$ in sec. $F_{\alpha}$ is given by Eq. 19.

The energy balance, Eq. 24, the time delays associated with the alpha power, Eq. 42 and the feedback system, Eq. 43 and equations 48,49 describing the density evolution are coupled to obtain the following system of ordinary differential equations.

$$
\begin{aligned}
\frac{d T}{d t} & =\mathcal{G}\left(T, n, f_{d}, P_{\alpha}(n, T), P_{\alpha}\left(T_{d}\right)\right) \\
\frac{d P_{\alpha}}{d t} & =\frac{1}{\tau_{\alpha}}\left[Q_{\alpha}(n, T)-P_{\alpha}(n, T)\right] \\
\frac{d T_{d}}{d t} & =\frac{1}{\tau_{d}}\left[T-T_{d}\right]
\end{aligned}
$$




$$
\begin{aligned}
\frac{d n}{d t} & =\mathcal{J}\left(T, n, S, f_{d}\right) \\
\frac{d f_{d}}{d t} & =\mathcal{K}\left(T, n, S, f_{d}, f_{s, d}\right)
\end{aligned}
$$

where the form of the functionals $\mathcal{G}, \mathcal{J}$, and $\mathcal{K}$ are given by Eqs. 24, 48, and 49 respectively. $P_{a}\left(T_{d}\right)$ is given by Eq. 38. $P_{\alpha}(T)$ is the alpha power absorbed by the plasma at time $t, Q_{\alpha}$ represents the amount of alpha power produced at time $t$. The parameters $\tau_{\alpha}$, and $\tau_{d}$ represent the delay times associated with the alpha power, and the feedback system.

In the next section the behavior of an auxiliary power control system are investigated by solving Eqs. 50 . 


\section{Results}

The Compact Ignition Tokamak (CIT), whose parameters are given in table 1 , is designed to achieve ignition (i.e. alpha particle dominated operation) in order to determine the effectiveness of alpha particle heating and its impact on confinement.

The results are presented in two parts. First, the heating sequence is examined and the time required to reach the desired operating temperature is compared to the pulse length. In the second part operation about the equilibrium temperature is considered and the auxiliary power control system is evaluated.

\subsection{Temperature Evolution}

During an ignition sequence in CIT the plasma temperature must evolve, from $T=0$ at the beginning of the discharge, to the desired operating temperature, $T_{S}$, in a time significantly shorter than the specified pulse length. This is a significant problem in CIT because of the short pulse length, and thus the shot must evolve in such a way so that the time to reach the temperature $T_{S}$ is minimized. This requires that the maximum amount of auxiliary power is supplied to the plasma and that the density evolves in such a way so that the plasma evolves through the Cordey pass. In this analysis the plasma temperature evolves from zero at the beginning of the current flat-top to the desired temperature some time during the flat- top. The maximum amount of auxiliary power available is $30 \mathrm{MW}$ (note that this is the power coupled to the plasma). For a conservative estimate we assume that a maximum of 25 MW can be coupled to the plasma. The results shown here, unless otherwise indicated are based on Goldston H-mode confinement.

Fig. 11.1 shows a CIT POP-CON plot under Goldston H-mode scaling. In this space the final operating point is denoted by point $\mathrm{A}$ which corresponds to density of $6 \times 10^{20} / \mathrm{m}^{3}$ and a temperature of $15 \mathrm{keV}$. 


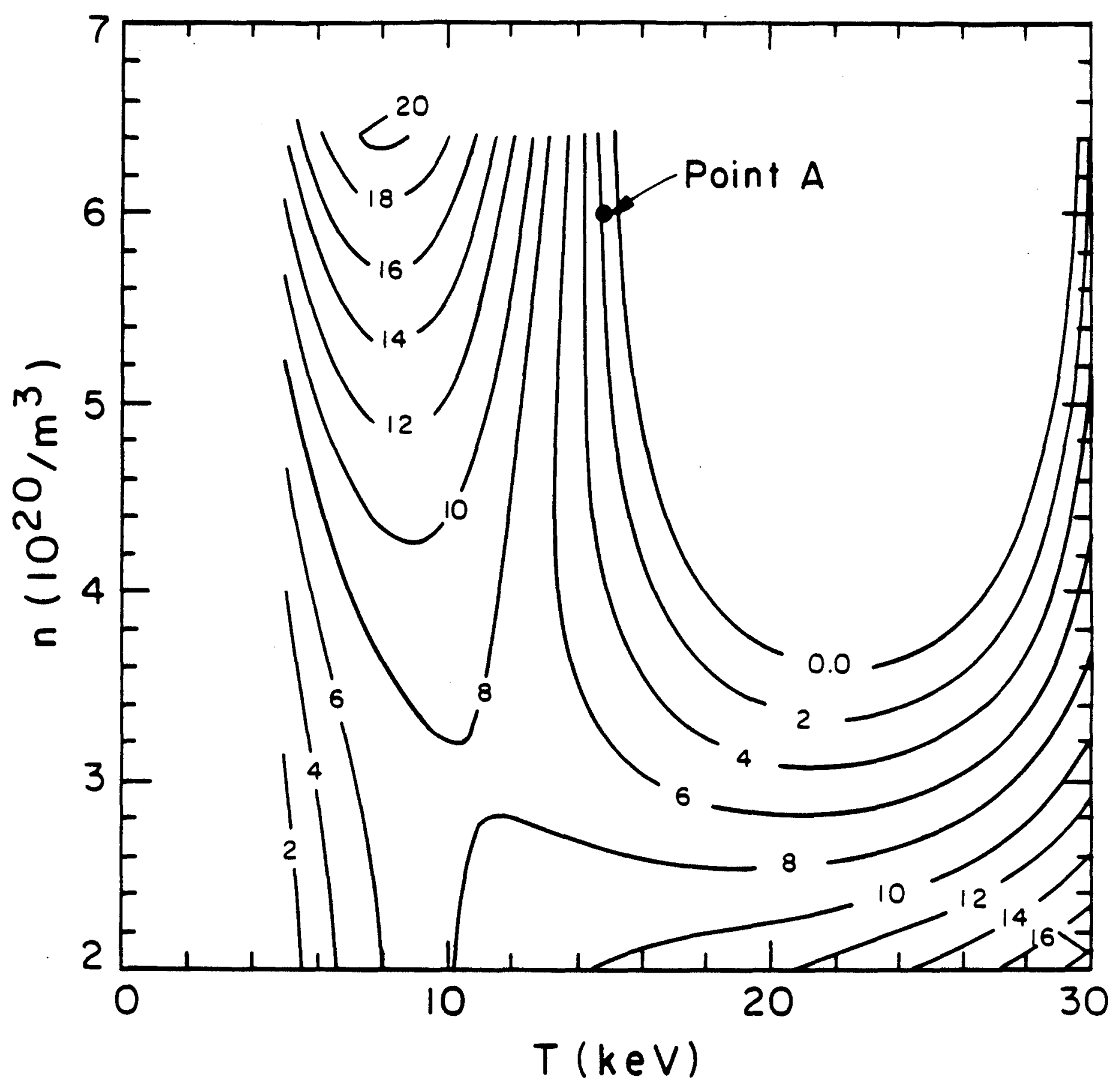

Figure 14: CIT POP-CON under Goldston H-mode scaling showing the desired final operating point (point $A$ ). 
Note, from Fig 11.1, that the Cordey pass occurs at a density of $\sim 3.0 \times$ $10^{20} / \mathrm{m}^{3}$ and a temperature of $\sim 11 \mathrm{keV}$. Here the density is varied with temperature as follows. For low temperatures and until the Cordey pass temperature is reached the density is constant and equal to $3 \times 10^{20} \mathrm{~m}^{3}$. For higher temperatures the density is ramped linearly between the Cordey pass temperature and the final operating temperature. The auxiliary power supplied to the plasma must be modulated if steady state operation is desired at $15 \mathrm{keV}$. From Fig. 11.1 we see that for steady state operation at a density of $6.0 \times 10^{20} / \mathrm{m}^{3}$ and a temperature of $15 \mathrm{keV}, 0.7 \mathrm{MW}$ of auxiliary power is required. Thus the power must be reduced from its maximum of $25 \mathrm{MW}$ to its steady state value of $0.7 \mathrm{MW}$ as the operating point is approached.

The plasma temperature, density, and the alpha, ohmic, auxiliary, radiation, and conduction powers are shown in Figs. 15, 16, and 17 for $\lambda=2$, $T_{1}=11 \mathrm{keV}$, and $T_{2}=16 \mathrm{keV}$. Under these conditions the plasma evolves to the final temperature in 2 seconds. The remaining 3 seconds during the current flat-top is used for physics and engineering operation at the high temperature equilibrium. In order for the plasma density to evolve as shown in Fig. 16 the fueling conditions shown in Fig. 18 must be provided. 'The result shown in Fig. 18 were obtained by assuming that the plasma evolves through a sequence of quasistatic equilibria and thus the $d n / d t$ term was neglected in the calculation of the source rate $S$. As seen from Fig. 18 strong fueling is required if the plasma is to evolve according to the above assumptions. Although, delivering the actual number of particles per second indicated in Fig. 18 is not difficult with present day pellet injection technology, pellet penetration will be difficult in a plasma of such high temperatures.

The plasma evolution presented above results in a high $Q$ operation as shown in Fig. 19. However, operation at the steady state equilibrium is constrained by stability considerations. These set a limit on the maximum $Q$ achievable and is discussed next. 


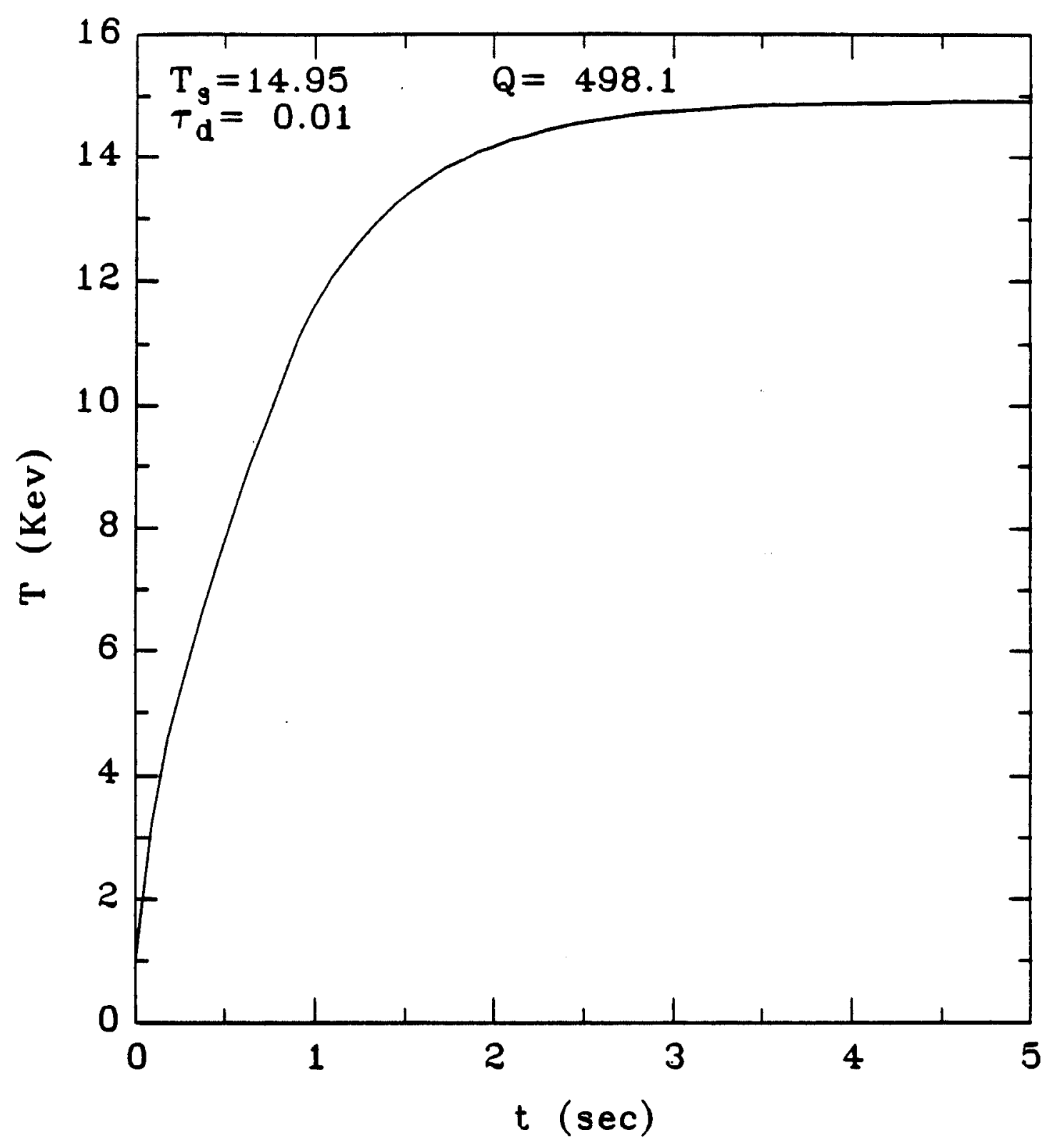

Figure 15: Time evolution of temperature for CIT under Goldston H-mode confinement. 


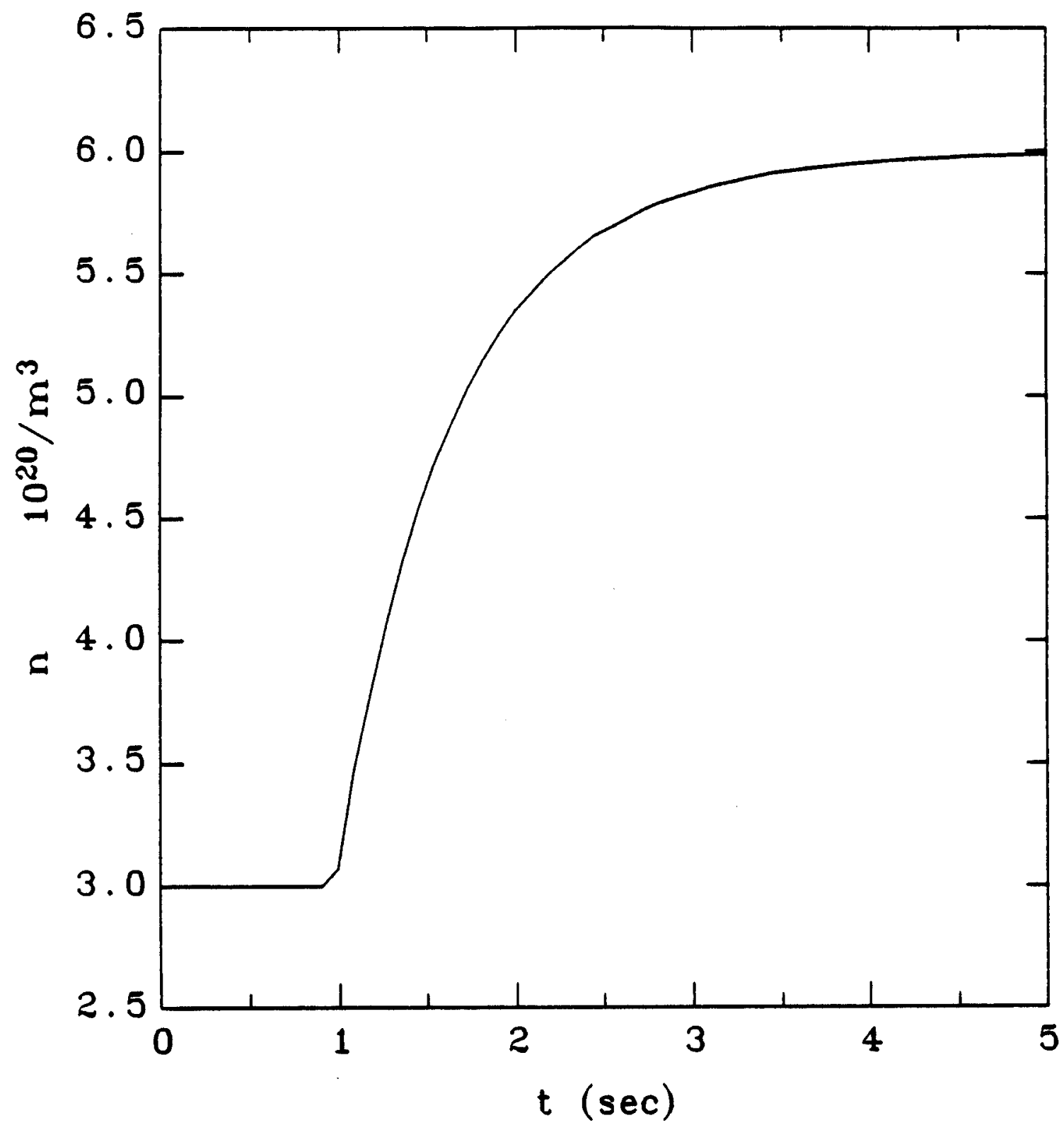

Figure 16: Time evolution of density for CIT under Goldston H-mode confinement. 


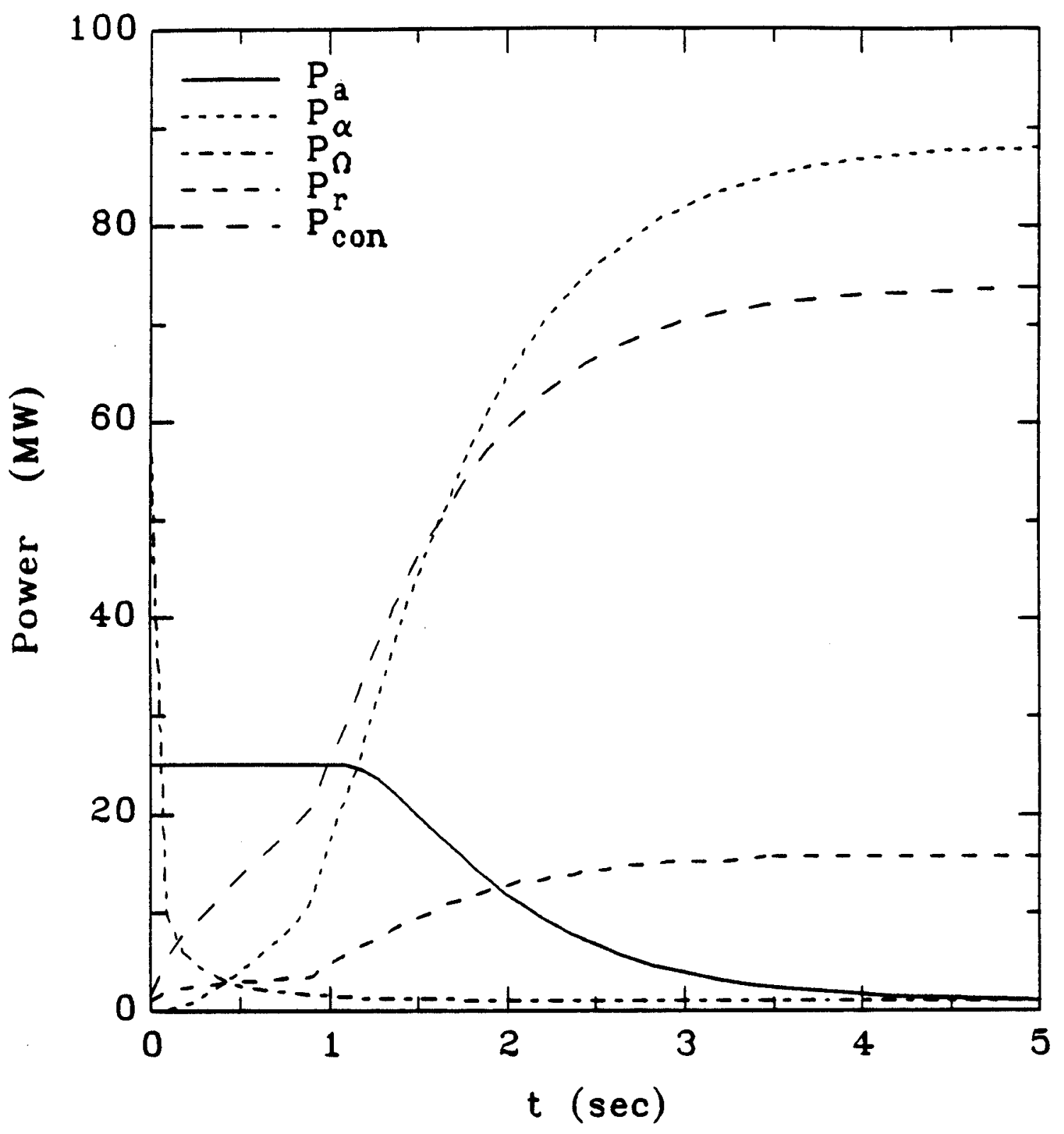

Figure 17: Time evolution of plasma powers for CIT under Goldston H-mode confinement. 


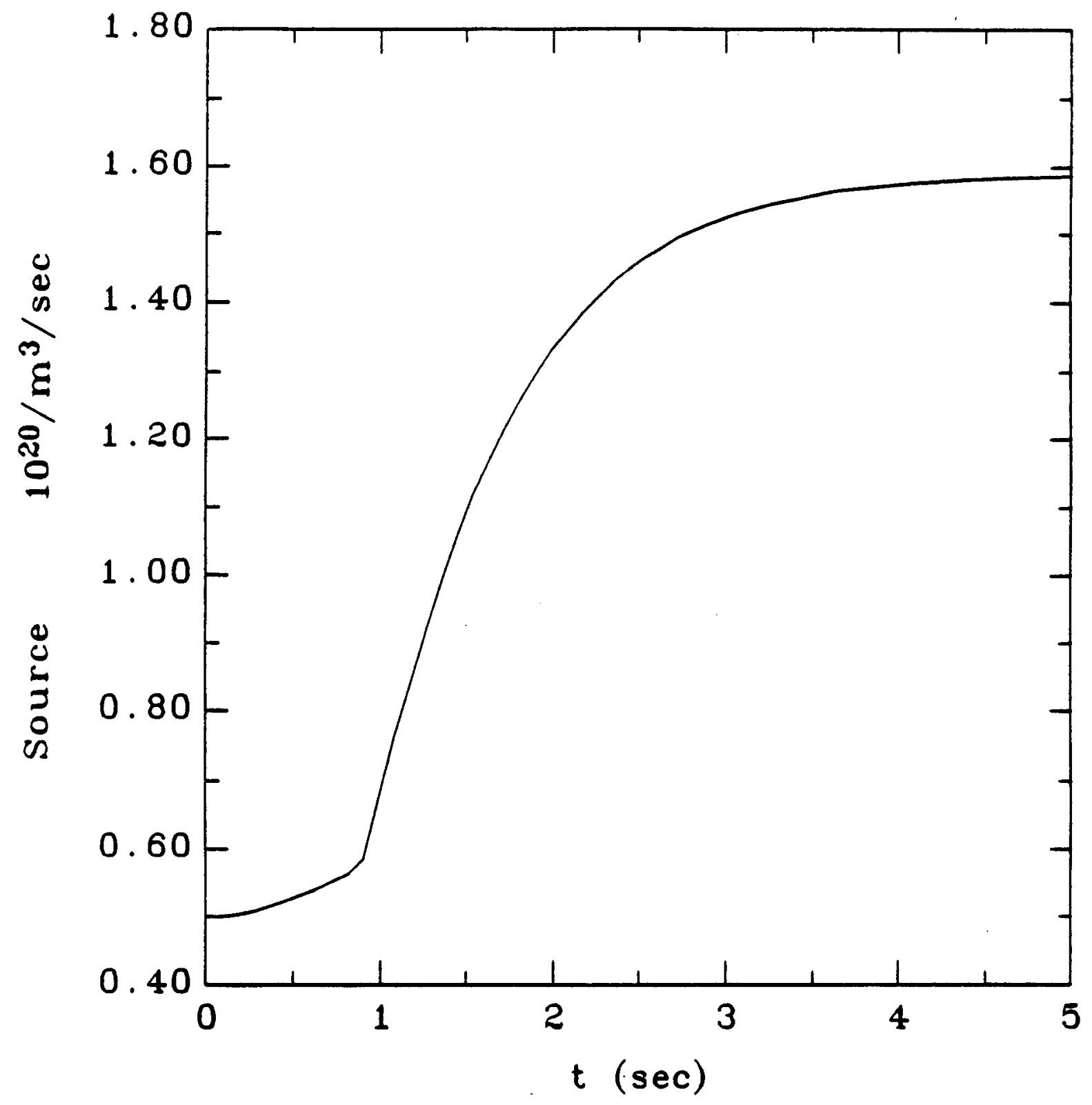

Figure 18: The particle source rate required to maintain the density evolution shown in Fig. 16 


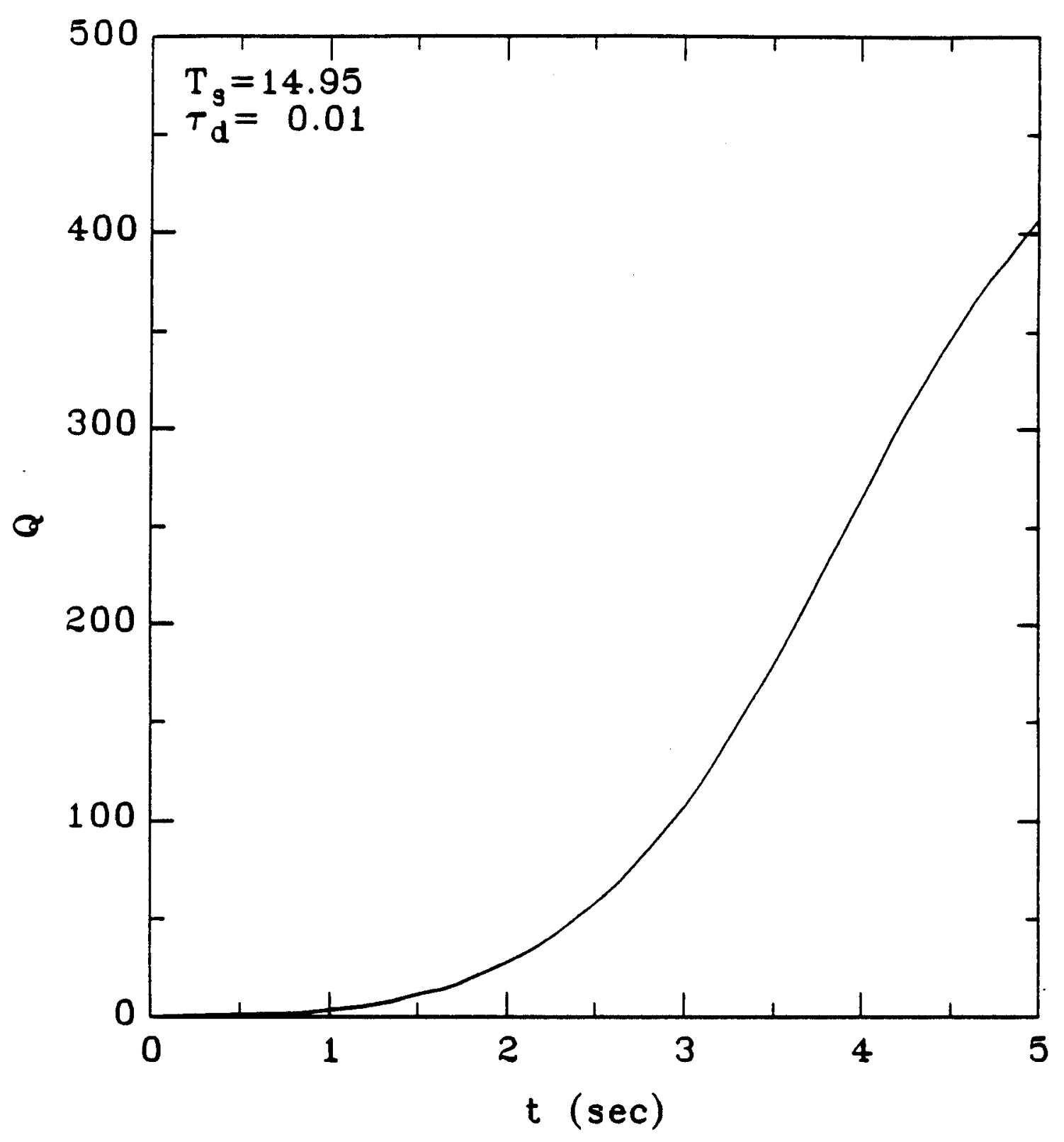

Figure 19: Time evolution of the thermonuclear $Q$ corresponding to Fig. 15 


\subsection{Burn Control}

Once the plasma has evolved to the desired operating point, steps must be taken to ensure stable operation. Temperature evolutions of the type presented in the previous section (Fig. 15) result in the formation of unstable equilibria. By modulating the amount of steady state auxiliary power supplied to the plasma, temperature deviations about the equilibrium can be effectively stabilized. The major issues investigated are: the magnitude of the run-away times, obtained by linear expansion about the equilibria, under different confinement relations, the relation between the maximum temperature to be stabilized and the steady state thermonuclear $Q$, the effect of the feedback delay time, $\tau_{d}$, on the maximum temperature deviations that can be effectively stabilized, the distinction between underdamped and critically damped systems, and the filtering of benign temperature fluctuations, such as sawteeth, from the feedback systern.

For effective stabilization of perturbations about an equilibrium temperature the feedback system must respond in a time faster than the runaway time $\tau_{R}$ of the instability. In Fig. 20 the e-folding time is shown as the runaway time for CIT under Goldston H-mode confinement scaling. Comparison of Fig. 20 with Fig. 21, which corresponds to Neo-Alcator scaling, shows the strong dependance of $\tau_{R}$ on confinement scalings. For example, at a density of $6 \times 10^{20} / \mathrm{m}^{3}$ and a temperature of $10 \mathrm{keV}$ the linear growth rate is $0.4 / \mathrm{sec}$ for Goldston and $2.0 / \mathrm{sec}$ for Neo- Alcator. This implies that the e-folding time for Neo-Alcator scaling is 5 times that for Goldston scaling.

As mentioned in section 9 the maximum temperature deviation that can be stabilized, in a system without time delays, is the difference between the operating temperature, $T_{S}$, and the equilibrium temperature at which the auxiliary power is zero, $T_{B}$. This maximum deviation is denoted by $\Delta T_{\max }=$ $T_{B}-T_{S}$ as seen in Fig. 13. The $Q$ value obtained at the steady state operating temperature, $T_{S}$ decreases as $\Delta T_{\max }$ increases. Fig. 22 shows the dependance 


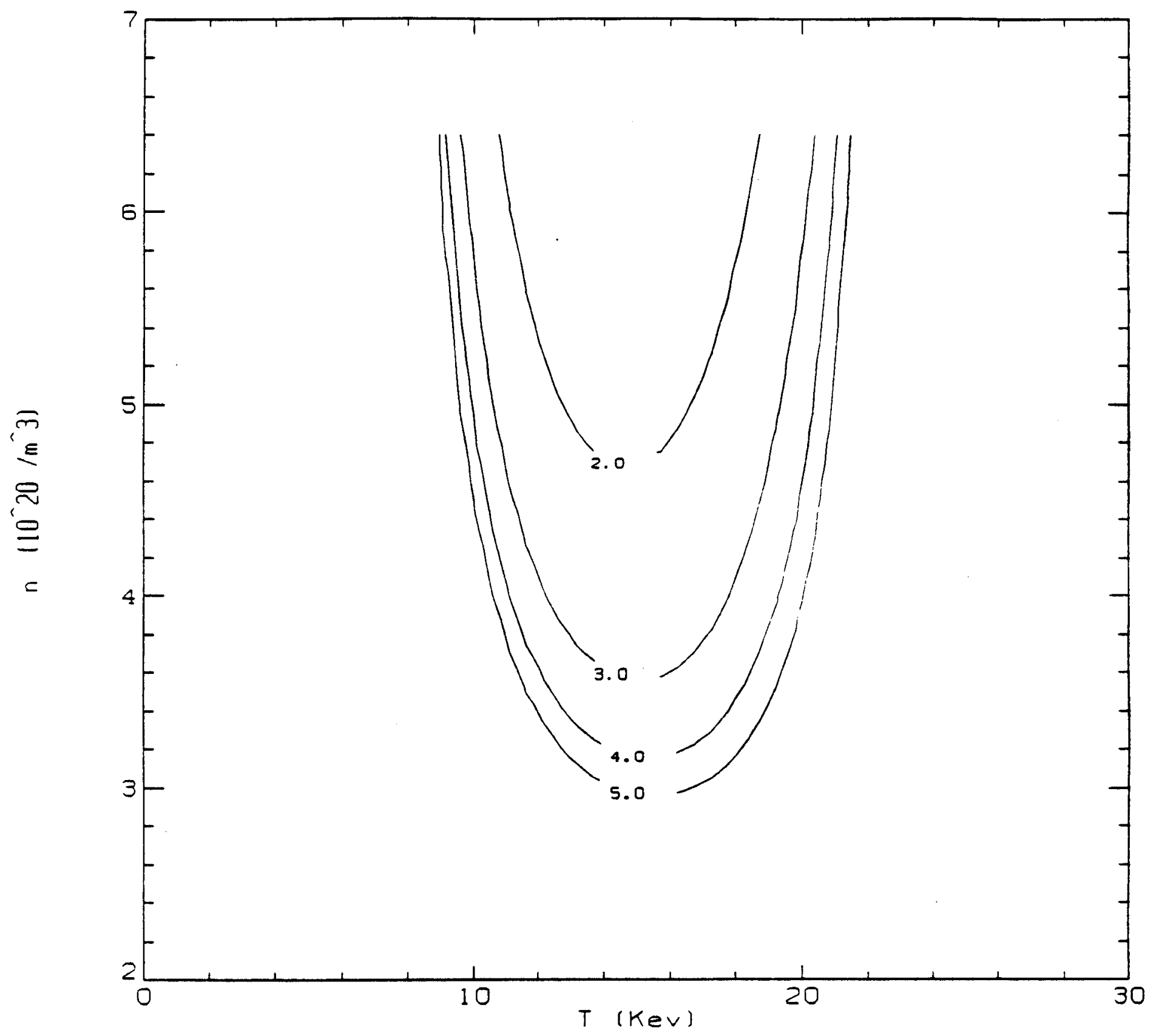

Figure 20: The e-folding time for the CIT unstable equilibria corresponding to Goldston $\mathrm{H}$-mode confinement 


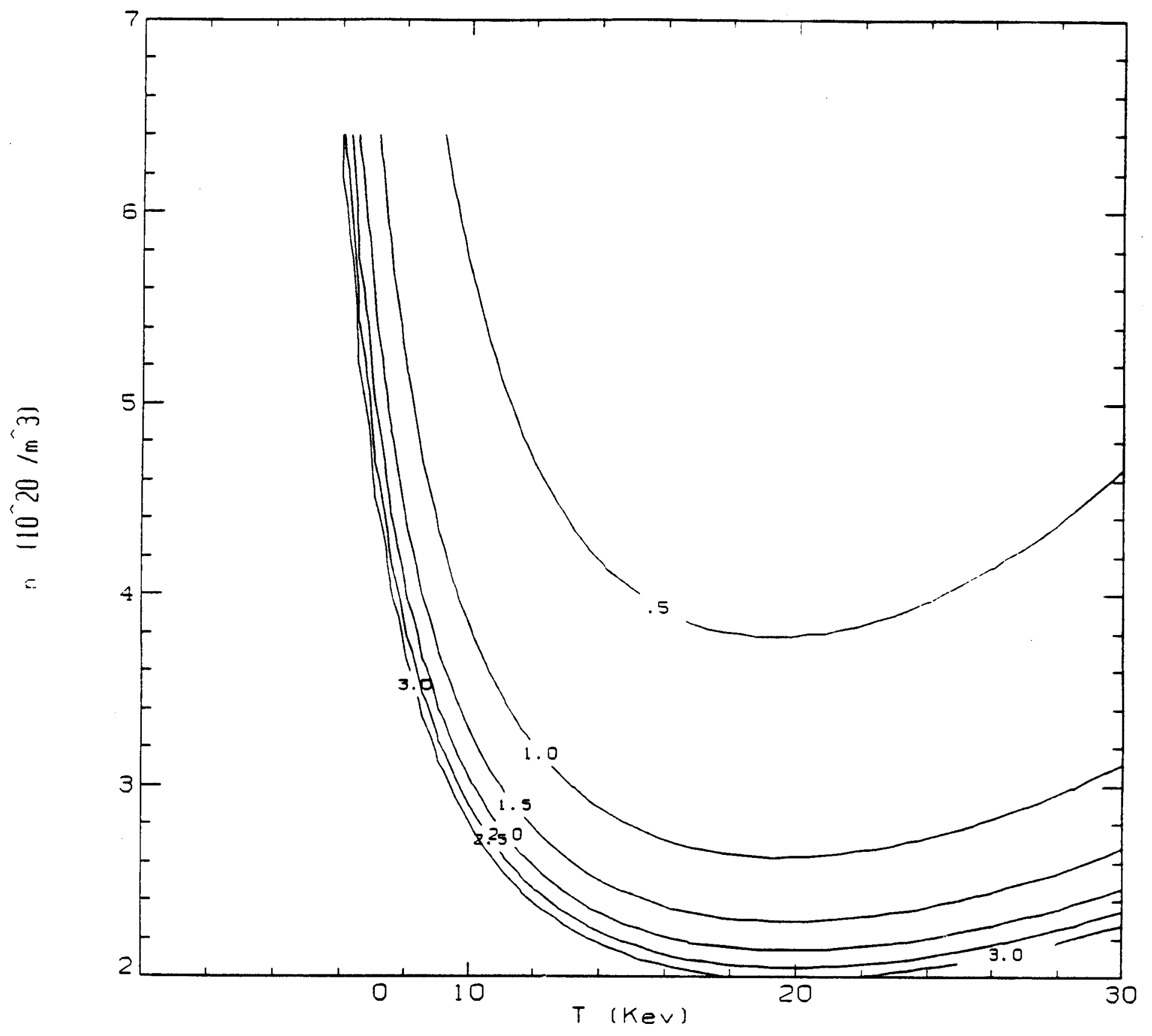

Figure 21: The e-folding time for the CIT unstable equilibria corresponding to Neo-Alcator confinement 


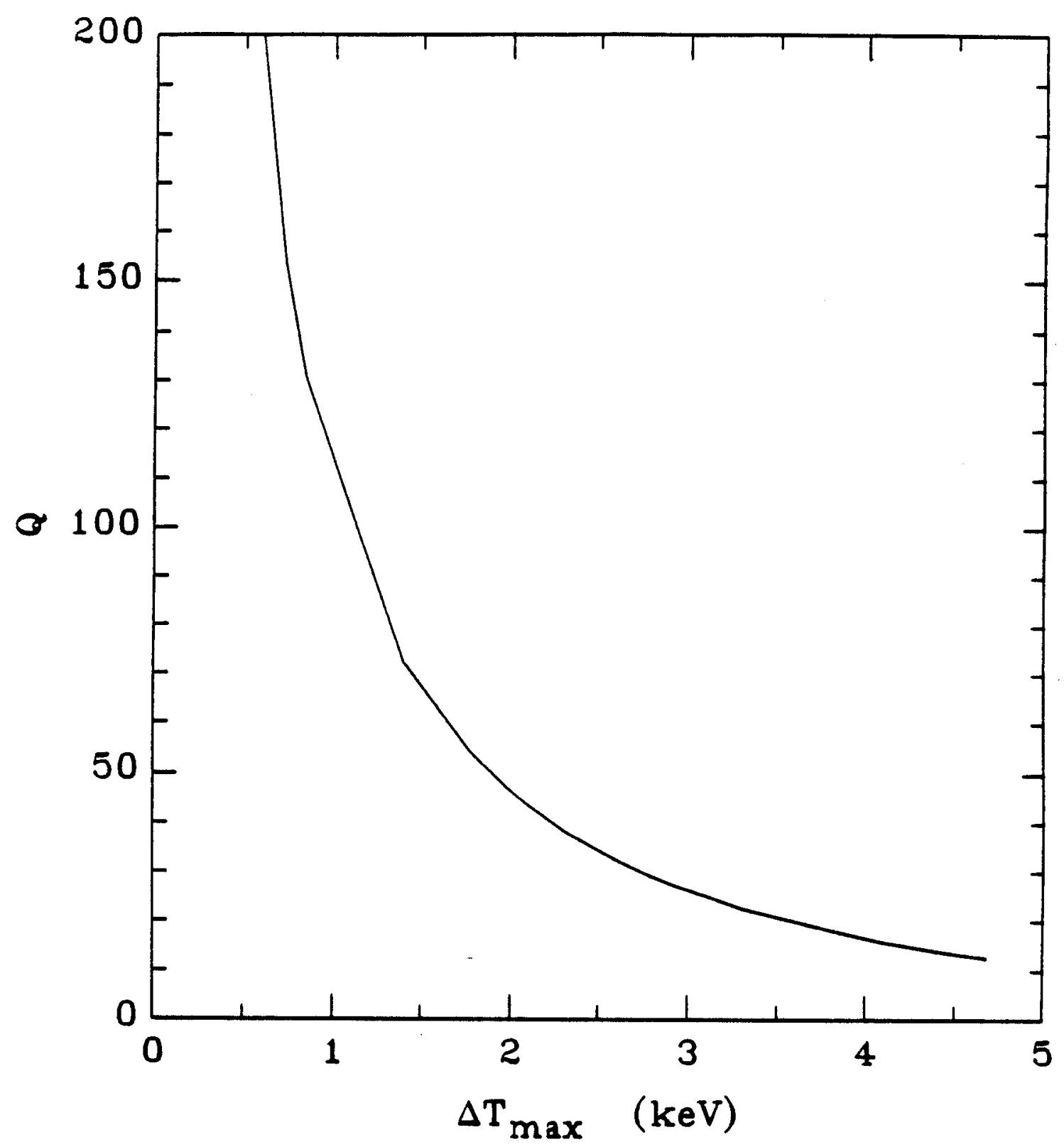

Figure 22: The dependance of the steady state $Q$ on the maximum temperature deviations stabilized in an ideal system 
of $\mathrm{Q}$ on $\Delta T$ for CIT under Goldston H-mode confinement. At this point an assessment of the maximum positive temperature deviations expected can give an estimation of the maximum achievable Q. For example, by assuming that the largest positive temperature perturbation to be expected is $1.5 \mathrm{keV}$, then the maximum $Q$ at which stable operation can be obtained is $\sim 65$.

Positive temperature fluctuations evolve with a characteristic time, $\tau_{R}$, while negative fluctuations can happen instantaneously (for example the introduction of impurities into the plasma). Therefore, the auxiliary power control system will have to respond faster to negative fluctuations than to positive ones. However, here we will consider the stabilization of instantaneous positive and negative fluctuations since such a case represents the most demanding scenario.

The feedback system, including the diagnostic system and the auxiliary power delivery system is characterized by the delay time $\tau_{d}$. For a certain feedback law, the maximum positive fluctuations to be stabilized as well as the maximum steady state $Q$ depends on the magnitude of $\tau_{d}$. By assuming a feedback law of the form given by Eq. 38 with $T_{1}=11.0, T_{2}=14.0$ and $\lambda=2.0$ the stabilization of an instantaneous $1.5 \mathrm{keV}$ positive temperature fluctuation is shown in Fig. 23, under three different values of the feedback delay time $\tau_{d}$. For $\tau_{d}=0.4$ the system resembles an underdamped oscillator. As $\tau_{d}$ decreases the system becomes more "damped" and the return to equilibrium becomes monotonic. For a certain feedback law the system will, as $\tau_{d}$ decreases pass through the "critically damped state" at which the return to equilibrium is the fastest. In Fig. 23 the curve corresponding to $\tau_{d}=0.12$ resembles a critically damped system and the approach to equilibrium is the fastest.

The model as described by Eqs. 50 is governed by three characteristic times, and they are: the alpha particle thermalization time, $\tau_{\text {ae }}$, the feedback delay time, $\tau_{d}$, and the energy confinement time, $\tau_{E}$. The effect of all these times is shown in Fig. 23. Due to the alpha particle thermalization the 


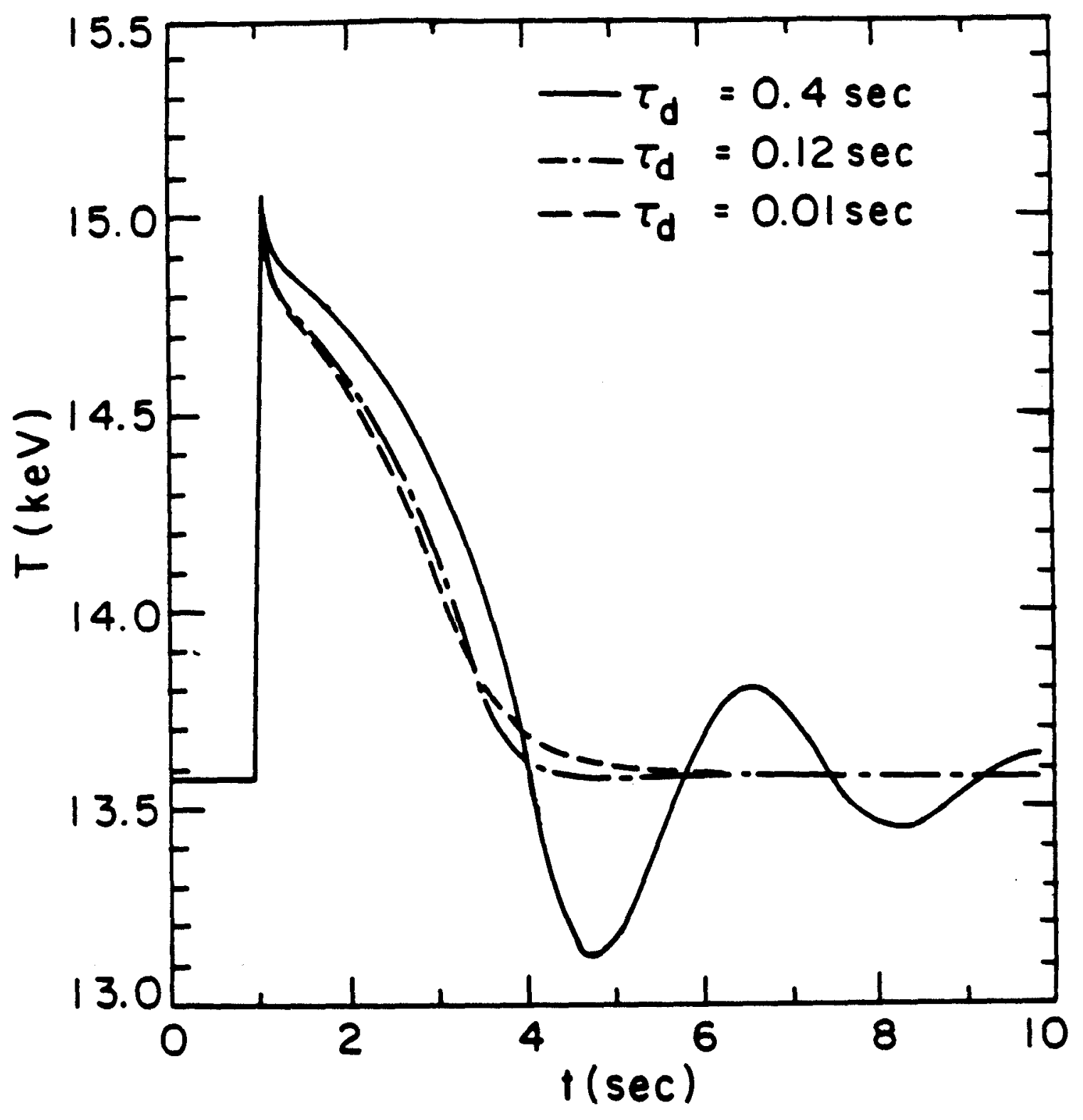

Figure 23: Stabilization of an instantaneous $1.5 \mathrm{keV}$ positive temperature deviation for different values of $\tau_{d}$ 
amount of alpha power deposited in the plasma after the temperature has increased by $1.5 \mathrm{keV}$ is equal to the amount of alpha power produced at the equilibrium for a time equal to the alpha particle thermalization time, $\tau_{\text {ae }}$. For a temperature of $13.57 \mathrm{keV}$, and a density of $6 \times 10^{20} / \mathrm{m}^{3} \mathrm{Eq} .37$ gives $\tau_{\alpha e}=0.05 \mathrm{sec}$. Since this value of $\tau_{\alpha e}$ is much shorter than the energy confinement time the temperature will drop rapidly immediately following the perturbation. Due to the feedback delay time $\tau_{d}$ the amount of auxiliary power supplied to the plasma will not be altered until a time $\tau_{d}$ later. The evolution of auxiliary power associated with the stabilization of the positive perturbations shown in Fig. 23 is given in Fig. 24.

For a negative temperature perturbation, as shown in Fig. 25, the amount of alpha power deposited in the plasma immediately after the temperature drop corresponds to the amount produced at the equilibrium and thus it tends to increase the plasma temperature. Therefore, the alpha particle thermalization time has a stabilizing effect on both positive and negative temperature fluctuations. Furthermore, the effect of the auxiliary power will not be seen until a time $\tau_{d}$ later and thus the temperature will decrease further after the alpha particle thermalization effect is observed, as it is clearly seen for the case of $\tau_{d}=0.4 \mathrm{sec}$. The evolution of auxiliary power associated with the stabilization of the negative perturbations shown in Fig. 25 is given in Fig. 26. The auxiliary power ramping required for stabilizing negative perturbations should be compatible with the specifications of the auxiliary power system. Large negative temperature deviations will require the supply of a large amount of auxiliary power within a very short time interval. For example note that a ramping of $20 \mathrm{MW} / \mathrm{sec}$ is required for stabilizing the 1.5 $\mathrm{keV}$ fluctuation in $1.5 \mathrm{sec}$.

As $\tau_{d}$ increases the system becomes more and more underdamped and eventually large oscillations develop and the system becomes unstable to every fluctuation. Fig. 27 illustrates a simulation in which an attempt is 


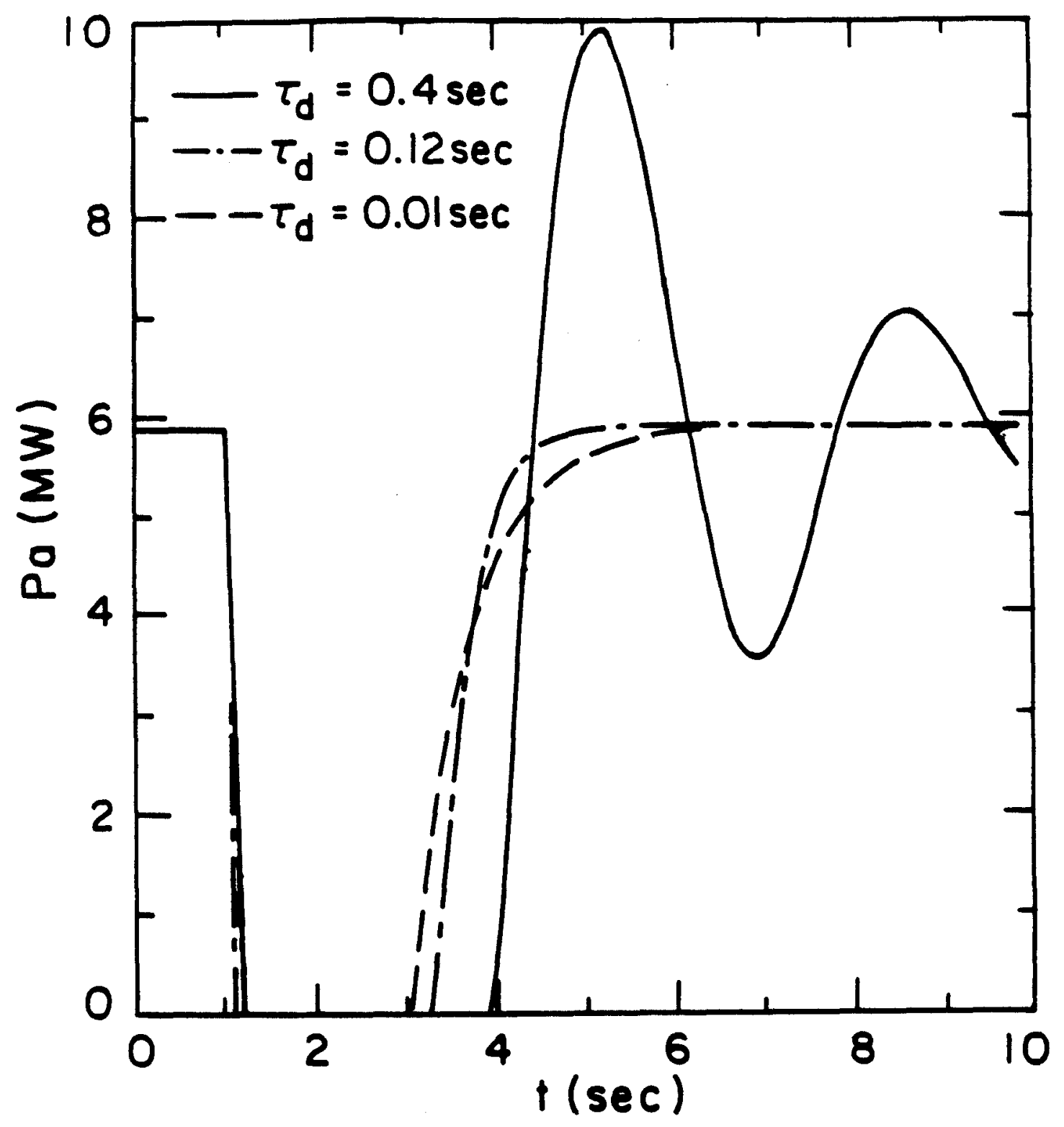

Figure 24: Evolution of auxiliary power required to stabilize a $1.5 \mathrm{keV}$ positive temperature deviation for various values of $\tau_{d}$. 


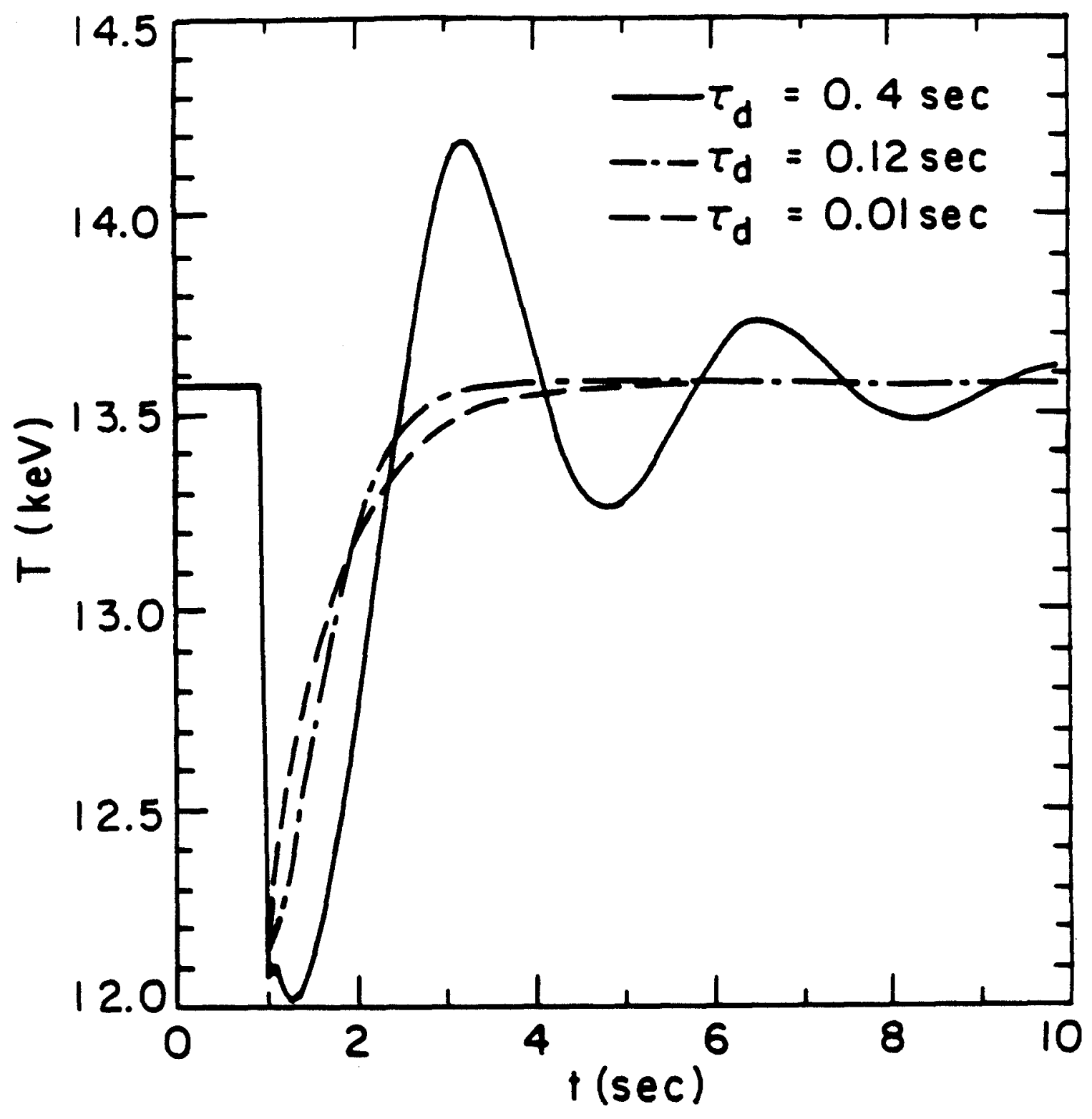

Figure 25: Stabilization of an instantaneous $1.5 \mathrm{keV}$ negative ternperature deviation for different values of $\tau_{d}$ 


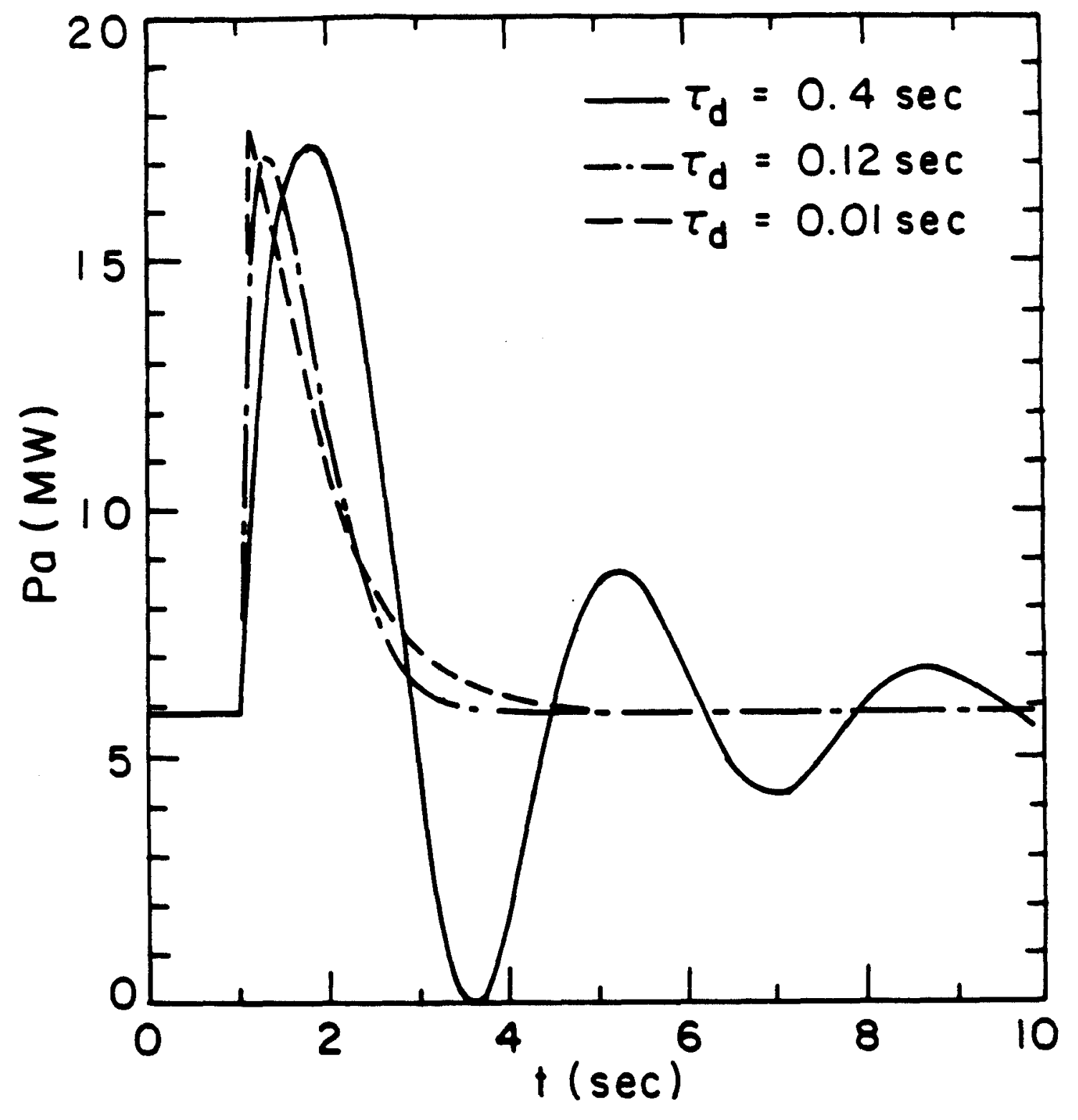

Figure 26: Evolution of auxiliary power required to stabilize a $1.5 \mathrm{keV}$ negative temperature deviation for various values of $\tau_{d}$. 


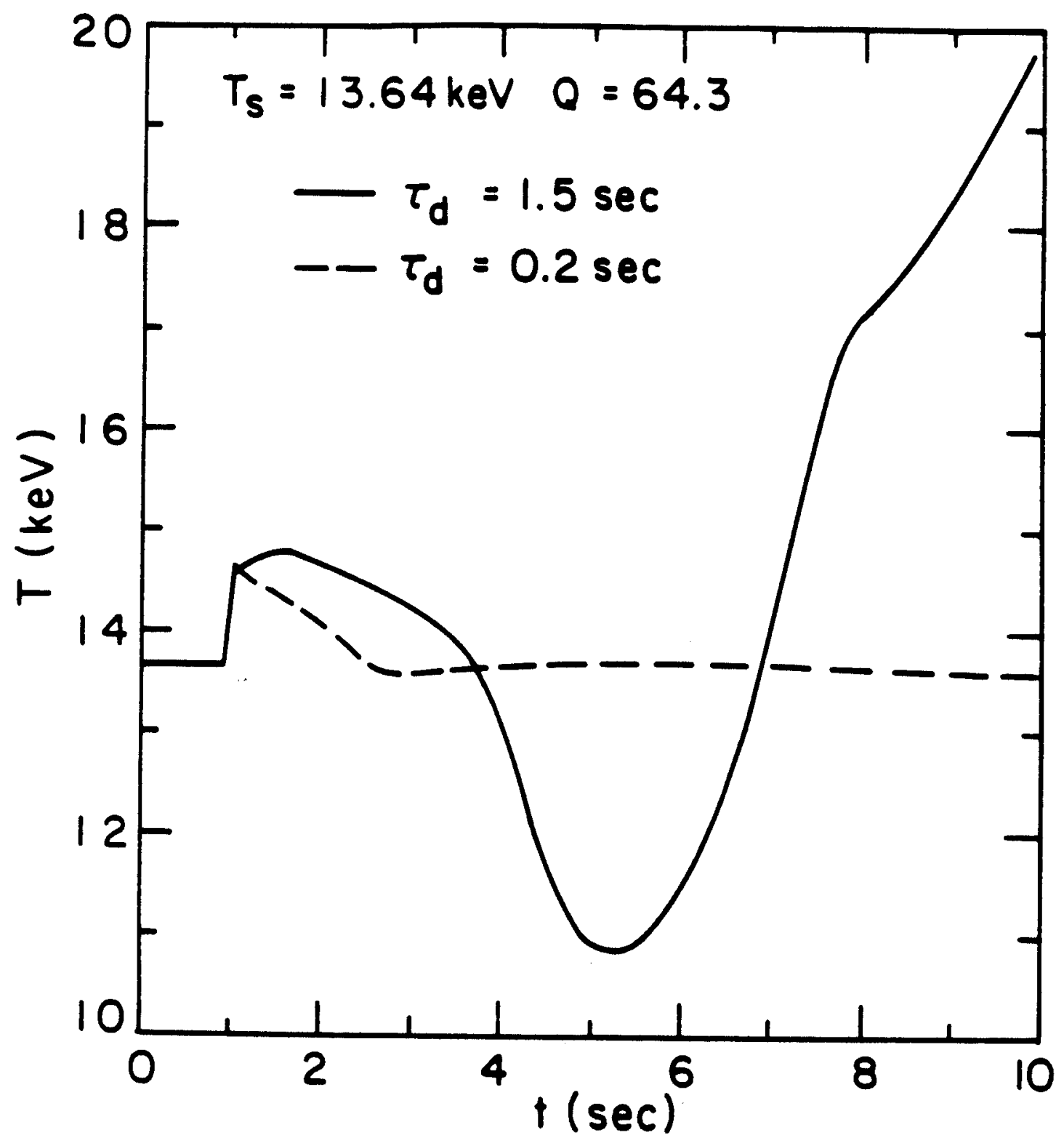

Figure 27: Simulation of a control system characterized a large delay time (solid line) and a small delay time (dashed line) 


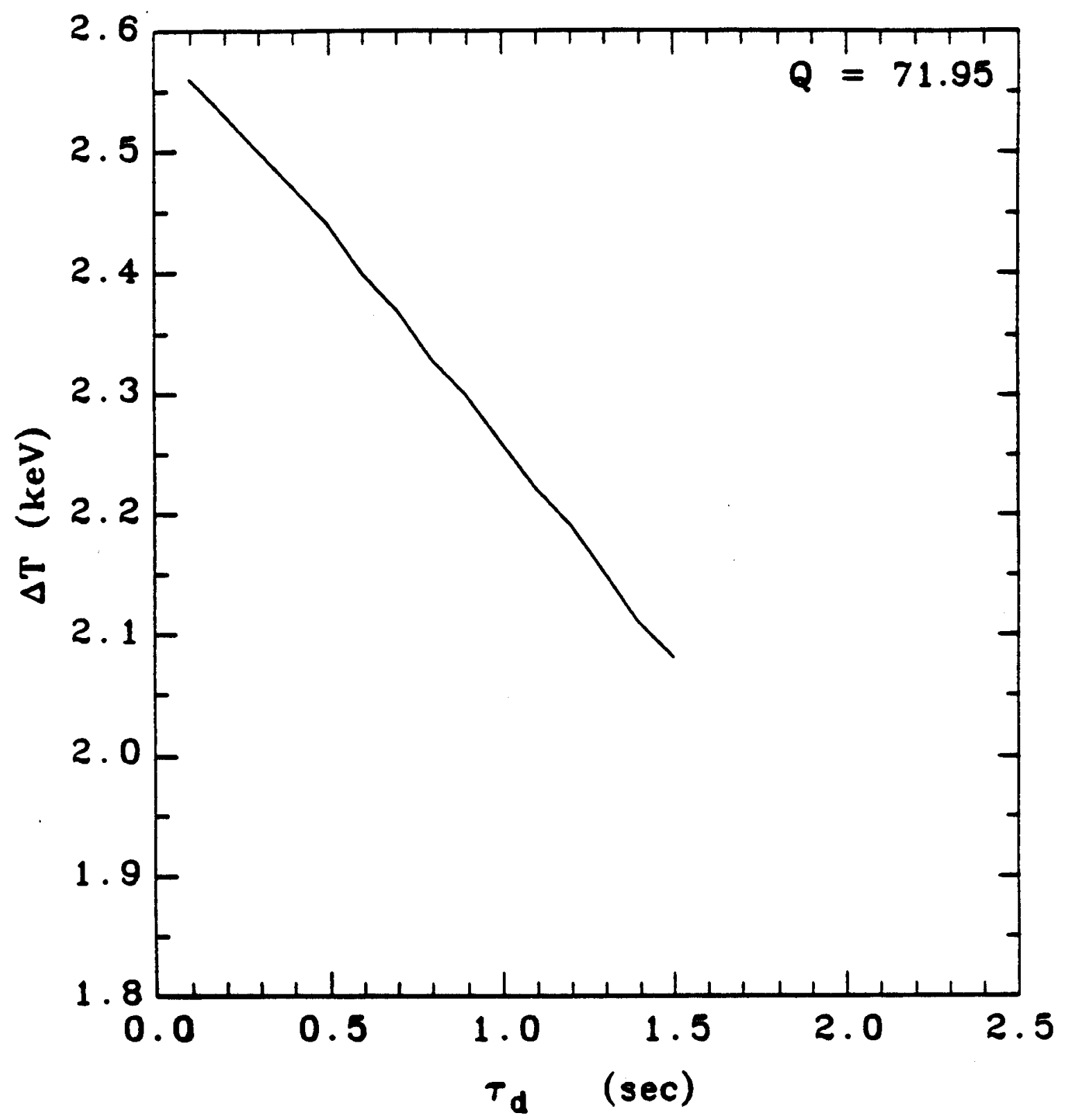

Figure 28: The maximum temperature deviation to be stabilized as a function of the feedback delay time $\tau_{d}$ 
made to stabilize a $1.5 \mathrm{keV}$ positive temperature fluctuation for $\tau_{d}=1.5 \mathrm{sec}$ (large delay time) and for $\tau_{d}=0.2 \mathrm{sec}$ (small delay time). Note that the system characterized by the large feedback delay time can not be stabilized.

In Fig. 28 the maximum temperature to be stabilized is plotted as a function of $\tau_{d}$, for operation at $Q=72$. In this case no temperature deviation can be stabilized for $\tau_{d}>1.5 \mathrm{sec}$.

The feedback system should be able to distinguish and filter all the benign temperature fluctuations that a plasma experiences as well as the noise introduced by the diagnostics. This is done by signal averaging. The time over which the averaging is done is a component of the overall feedback delay time $\tau_{d}$. For example in order to filter the temperature perturbations introduced by sawteeth the averaging must be done over a certain number of sawteeth periods. However, since the exact period of sawteeth is not known the system has to be designed so that it stabilizes over a wide range of periods without violating the maximum limit on $\tau_{d}$. A complete study of this phenomenon requires the development of a sawtooth model in the context of one dimensional transport. Such a model is presently under development at MIT.

\section{Conclusions}

Auxiliary power modulation can be used for operating CIT. By supplying auxiliary power to the plasma and appropriately modulating it he tokamak operating space can be mapped, the desired operating point can be reached, and control about the operating point can be achieved. It has been estimated that stable operation is possible with $Q \sim 65$. Large delay times result in a system resembling an underdamped oscillator as it returns to equilibrium after a temperature fluctuation. Feedback systems characterized by delay times in the order of $0.1-0.3$ seconds can stabilize temperature fluctuations of $1.5 \mathrm{keV}$ at $Q \sim 65$. Benign temperature fluctuations are filtered by signal averaging over a specified time period which is compatible with the overall maximum feedback delay time. 


\section{APPENDIX A}

$$
\begin{aligned}
K_{l} & =\frac{1.2 \times 10^{5}}{1+\nu_{n}+\nu_{T}} \\
K_{\Omega} & =\frac{1.67 \times 10^{4}}{1+1.5 \nu_{T}}\left(\frac{1+\kappa^{2}}{\kappa}\right)^{2} Z_{\text {eff }} \\
K_{\alpha} & =\frac{5.6 \times 10^{5}}{\nu_{T}} \\
K_{\mathrm{b}} & =\frac{5.3^{3}}{1+2 \nu_{n}+.5 \nu_{T}} Z_{\text {eff }} \\
K_{a} & =\frac{5.07 \times 10^{4}}{\kappa} \\
C_{1} & =\frac{0.0507}{\kappa^{.5}} \\
C_{2} & =\frac{.14}{\nu_{T}} \kappa^{.5} \\
f & =\frac{n_{e, 0}}{n_{e}} \approx\left(1+1.24 \nu_{n}\right)^{.502}
\end{aligned}
$$

where $\bar{n}_{e}$ is the line averaged electron density. 


\section{Acknowledgements}

This work was performed by the U. S. Department of Energy contract numbers DE-AC02-78ET51013, and DE-AC02-76CH03073. 


\section{List of Figures}

1 Plot of the rate of change of temperature with time, $\dot{T}$, vs temperature $T$. . . . . . . . . . . . . . 10

2 A plot showing the original system (solid line) and the ignited system with $P_{a}=P_{I}$ (dotted line). .......... 11

3 General plasma operating contours of auxiliary power showing the marginal ignition ridge (MIR) contour (dotted line). . . . 13

4 CIT plasma operating contours of auxiliary power for Goldston $\mathrm{H}$-mode scaling . . . . . . . . . . . . . 15

$5 \mathrm{n}$ - $\mathrm{T}$ plot with contours of linear growth rates $(\gamma)$ of plasma temperature corresponding to Fig. 4 . . . . . . . . 16

6 Location of the high temperature stable equilibria corresponding to zero auxiliary power under various confinement scalings. 18

$7 \quad Q$ at the marginal ignition ridge (MIR) as a function of plasma density for Neo-Alcator (solid line), Goldston (dotted line), and Kaye-Goldston (dashed line) confinement scalings . . . 22

8 The marginal ignition ridge (MIR) and the $Q=5$ contour are shown for CIT under Neo-Alcator scaling . . . . . . . . 23

9 The marginal ignition ridge (MIR) and the $Q=5$ contour are shown for CIT under Goldston H-mode scaling . . . . . . 24

10 The marginal ignition ridge (MIR) and the $Q=5$ contour are shown for CIT under Kaye-Goldston H-mode scaling . . . . . 25

11 Temperature evolution of an ignited CIT with "small" amount of auxiliary power. . . . . . . . . . . . . . 26

12 Temperature evolution of an ignited CIT with "large" amount of auxiliary power. . . . . . . . . . . . . . 27

13 Schematic representation of an original system (a), the form of the auxiliary power (b), and the final system indicating the steady state operating temperature $T_{S} \ldots \ldots . \ldots 38$ 
14 CIT POP-CON under Goldston H-mode scaling showing the desired final operating point (point A) . . . . . . . . . . 45

15 Time evolution of temperature for CIT under Goldston Hmode confinement. . . . . . . . . . . . . . . . . 47

16 Time evolution of density for CI'T under Goldston H-mode confinement. ................... . . 48

17 Time evolution of plasma powers for CIT under Goldston Hmode confinement. . . . . . . . . . . . . . . . . . . 49

18 The particle source rate required to maintain the density evolution shown in Fig. $16 \ldots \ldots 50$

19 Time evolution of the thermonuclear $Q$ corresponding to Fig. 1551

20 The e-folding time for the CIT unstable equilibria corresponding to Goldston $\mathrm{H}$-mode confinement . . . . . . . . . . . . 53

21 The e-folding time for the CIT unstable equilibria corresponding to Neo-Alcator confinement . . . . . . . . . . . . . . 54

22 The dependance of the steady state $Q$ on the maximum temperature deviations stabilized in an ideal system . . . . . . 55

23 Stabilization of an instantaneous $1.5 \mathrm{keV}$ positive temperature deviation for different values of $\tau_{d} \ldots \ldots . \ldots 57$

24 Evolution of auxiliary power required to stabilize a $1.5 \mathrm{keV}$ positive temperature deviation for various values of $\tau_{d} . \ldots 59$

25 Stabilization of an instantaneous $1.5 \mathrm{keV}$ negative temperature deviation for different values of $\tau_{d} \ldots \ldots . . \ldots 60$

26 Evolution of auxiliary power required to stabilize a $1.5 \mathrm{keV}$ negative temperature deviation for various values of $\tau_{d} \ldots \ldots 61$

27 Simulation of a control system characterized a large delay time (solid line) and a small delay time (dashed line) . . . . . . 62

28 The maximum temperature deviation to be stabilized as a function of the feedback delay time $\tau_{d} \ldots \ldots$. . . . . . . 64 


\section{References}

[1] L. Spitzer. Physics of Fully Ionized Gases. Interscience, New York, second edition, 1962.

[2] J.P. Freidberg. Ideal Magnetohydrodynamics. Modern Perspectives in Energy. Plenum Press, New York, 1987.

[3] R.R. Parker et. al. Progress in tokamak research at mit. Nuclear Fusion, 25(3), 1985.

[4] R. Goldston. Energy confinement scaling for tokamaks. Plasma Phus. Controlled fusion, 26:87, 1984.

[5] I.H. Hutchinson. Requirements for Ohmic Ignition. Journal of Fusion Energy, 6(3), 1987.

[6] International Atomic Energy Egency. International Tokamak Reactor, Zero Phase, Vienna, 1980.

[7] C. Powell. Control of the Energy Balance in a Fusion Plasma. Plasma Physics, 15:1007, 1973.

[8] E. L. Vold T. K. Mau and R. W. Cohn. Tokamak power reactor ignition and time-dependant fractional power operation. Fusion Technology, 12:197, 1987.

[9] T. W. Petrie and J. M. Rawls. Burn control resulting from toroidal field ripple. Nuclear Fusion, 20:419, 1980.

[10] T. W. Petrie and J. M. Rawls. Burn control via regulated ripple applied to reactor grade plasmas. Nuclear Fusion, 20:1461, 1980.

[11] T. E. Stringer. Effect of the magnetic field ripple on diffusion in tokamaks. Nuclear Fusion, 12:689, 1972. 
[12] K. C. Shaing and J. D. Callen. Neoclassical ripple transport in tokamaks. Nuclear Fusion, 22:1061, 1982.

[13] L. Bromberg D. R. Cohn. Passive thermal-stability control in ignited tokamaks by ripple transport and radial motion. Nuclear Fusion, 21:201, 1981.

[14] A. Sestero. Control of burn-up phase. In G. Casini, editor, Plasma Physics for Thermonuclear Fusion Reactors, page 389. 1981.

[15] M. Ohnishi A. Saiki and M. Okamoto. Space-dependent analyis of feedback control to suppress thermal runaway by compressiondecompression. Nuclear Technology/Fusion, 5:326, 1984.

[16] S. V. Putvinskii. Stability of thermonuclear burn in a tokamak. Sov. J. Plasma Physics, 6:694, 1980.

[17] U Carretta E. Minardi and N. Bacelli. Flasma position control in a tokamak reactor around ignition. Nuclear Fusion, 26:599, 1986.

[18] L. Bromberg J. L. Fisher D. R. Cohn. Active burn control of ignited plasmas. Nuclear Fusion, 20(2), 1980.

[19] L. Harten V. Fuchs and A. Bers. Creating stable tokamak reactor equilibria by supplemental heating. Nuclear Fusion, 20:833, 1980.

[20] V. Fuchs L. Harten and A. Bers. On tokamak thermal equilibria and thermal stability. Nuclear Fusion, 20(5), 1980. 